\title{
Liraglutide Improves Renal Endothelial Function in Obese Zucker Rats on a High-Salt Diet
}

\author{
Vijayakumar Sukumaran, Hirotsugu Tsuchimochi, Takashi Sonobe, Mikiyasu Shirai, \\ and James T. Pearson

\begin{abstract}
Department of Cardiac Physiology (V.S., H.T., T.S., J.T.P.) and Center of Advanced Medical Research for Pulmonary Hypertension (M.S.), National Cerebral and Cardiovascular Center Research Institute, Suita, Osaka, Japan; and Department of Physiology, Monash Biomedicine Discovery Institute, Monash University, Clayton, Australia (J.T.P.)
\end{abstract}

Received November 2, 2018; accepted March 21, 2019

\begin{abstract}
Metabolic syndrome is a common risk factor in chronic kidney disease. We investigated whether liraglutide [(LIRA), a glucagonlike peptide-1 receptor (GLP-1R) agonist] treatment improved renal vascular function and renal remodeling in male Zucker rats on a high-salt diet $(6 \% \mathrm{NaCl})$. Zucker lean $(+/+)$ and obese $(\mathrm{fa} / \mathrm{fa})$ rats ( 8 weeks old) were treated with vehicle or LIRA $(0.1 \mathrm{mg} / \mathrm{kg}$ per day) for 8 weeks on a high-salt diet. The glomerular filtration rate (GFR) was measured at 0 and 8 weeks using the fluorescein isothiocyanate/sinistrin method in conscious rats. We used X-ray microangiography to measure renal arterial vessel diameter $(70-350 \mu \mathrm{m})$ and vessel number in vivo in anesthetized rats. Renal protein expression levels of nitrotyrosine, CD-68, endothelial nitric oxide synthase (eNOS), vascular endothelial growth factor (VEGF), transforming growth factor- $\beta 1$, cyclooxygenase-2, and GLP-1R were assessed by western blotting. Renal gene expressions were determined by
\end{abstract}

real-time polymerase chain reaction. In contrast to vehicletreated rats, fa/fa-LIRA rats improved GFR, nitric oxide (NO)mediated vasodilation in response to acetylcholine and sodium nitroprusside in small arterial vessels ( $<200 \mu \mathrm{m}$ diameter). LIRA treatment increased vessel responsivity to NO donors in comparison with vehicle treatment. Increases in the expressions of proinflammatory, profibrotic, and oxidative stress related genes in $\mathrm{fa} / \mathrm{fa}$ rats relative to $+/+$ were unaltered by LIRA, other than a trend toward attenuation of VCAM-1 gene expression. However, LIRA treatment increased protein expressions of eNOS $(P=0.014)$ and VEGF $(P=0.063)$, while reducing glomerular macrophage infiltration in comparison with vehicle-treated fa/fa rats. Low-dose LIRA treatment improved renal vascular function through amelioration of vascular dysfunction and improved NO-mediated dilation of small intrarenal arteries and arterioles and a reduction in renal inflammation.

\section{Introduction}

With the increasing prevalence of obesity in modern societies, and as a consequence of associated comorbidities, obesity is one of the leading global causes of cardiovascular morbidity and mortality. Obesity leads to insulin resistance, vascular oxidative stress, endothelial dysfunction, and consequently reduced availability of vascular nitric oxide (NO) and impaired dilation in the microcirculation of many vascular beds, which contributes to the development of multiple cardiovascular complications including chronic kidney disease (CKD) (Satoh, 2012; Rao et al., 2015). An increasing number of

Some of the findings from this study were part of an oral presentation entitled "Liraglutide treatment improves renal vascular function in Zucker rats as visualized by microangiography" in a symposium at the American Society of Nephrology (Kidney Week) meeting, October 30 to November 5, 2017, in New Orleans, LA.

This Research was supported financially as an investigator sponsored study by Novo Nordisk Pharma Ltd., an affiliate of Novo Nordisk A/S of Denmark. https://doi.org/10.1124/jpet.118.254821. the population that are obese and hypertensive develop CKD (Wu et al., 2012). In the obese population, salt-sensitive hypertension is strongly associated with the progression of target-organ damage and renal injury associated with glomerulosclerosis, inflammation, and albuminuria (Quigley et al., 2009; Wei et al., 2017). It has also been established that a high-salt diet directly affects function of the renal vascular system in part through elevated generation of reactive oxygen species (ROS) and inflammation, independent of blood pressure changes (de Cavanagh et al., 2010). Various experimental studies provide evidence that high-salt intake directly impacts the balance of production of $\mathrm{NO}$ and transforming growth factor- $\beta 1$ (TGF- $\beta 1$ ) (Sanders, 2009a; Patel et al., 2016). Elevated endothelial TGF- $\beta 1$ production contributes directly to the development of hypertension, which in a state of endothelial dysfunction and a proinflammatory and oxidative endothelial cell environment leads to diminished NO-mediated negative regulation of TGF- $\beta 1$ effects in the vasculature, and ultimately results in glomerular fibrosis and

ABBREVIATIONS: ACh, acetylcholine; BUN, blood urea nitrogen; CKD, chronic kidney disease; COX, cyclooxygenase; EDHF, endotheliumderived hyperpolarizing factor; eNOS, endothelial nitric oxide synthase; ET-1, endothelin-1; FN-1, fibronectin-1; GFR, glomerular filtration rate; GLP1, glucagon-like peptide-1; GLP-1R, glucagon-like peptide-1 receptor; LIRA, Liraglutide; Ln, lean; MAP, mean arterial pressure; NO, nitric oxide; NT, nitrotyrosine; ROS, reactive oxygen species; SNP, sodium nitroprusside; TGF- $\beta 1$, transforming growth factor- $\beta 1$; VEGF, vascular endothelial growth factor; Veh, vehicle. 
exacerbation of vascular dysfunction (Sanders, 2009a,b; Meng et al., 2010). Moreover, studies have shown that dietary salt intake also exacerbates insulin resistance, and metabolic syndrome could play a major role in renal function decline (Garg et al., 2011; Oh et al., 2015). The Zucker obese rat model has been shown to develop hypertension associated with impaired kidney function on a high-salt diet (Ali et al., 2015; Maheshwari et al., 2018). The combination of obesity and high-salt intake thus contributes to the acceleration of renal vascular dysfunction independent of blood pressure changes.

Glucagon-like peptide-1 (GLP-1) acts through the GLP-1 receptor (GLP-1R), which is a G-protein-coupled receptor expressed on coronary endothelial cells, cells of the gastrointestinal tract, vascular smooth muscle, proximal tubules, and glomeruli of the kidney (Ban et al., 2008). Recent work has shown that in the streptozotocin-treated rat and the diabetic nephropathy-prone KK/Ta-Akita mouse model treatment with a GLP-1R agonist, liraglutide (LIRA), has renoprotective effects (Hendarto et al., 2012; Fujita et al., 2014). This was primarily attributed to an antioxidant effect mediated through inhibition of NADPH oxidase, normalization of TGF$\beta 1$, and elevation of cAMP/protein kinase A levels (Hendarto et al., 2012; Fujita et al., 2014). Others have also reported that the protective effects of GLP-1 agonists in glomerular endothelial cells are mediated by inhibition of angiotensin II via AMP-dependent protein kinase-mediated cRaf phosphorylation (Mima et al., 2012). However, there are no in vivo studies that show the effects of sustained GLP-1 activation on functional changes in small renal arteries and arterioles, which play the most important role in regulating renal blood flow and pressure, or the effects on renal vascular remodeling. Diabetic nephropathy and CKD are often associated with arterial and glomerular arteriole hyalinosis (wall thickening with hyaline deposition) and endothelial damage (Yuzawa et al., 2008). Moreover, no studies that we are aware of, have determined whether GLP-1 agonist can ameliorate renal dysfunction in an experimental model of obesity combined with high-salt intake.

We have successfully developed and validated effective approaches to assess segmental vascular structure and function, and blood flow distribution in the kidney in rodents using in vivo microangiography with synchrotron radiation (Eppel et al., 2009; Shirai et al., 2013). Taking advantage of this laboratory X-ray system, we investigated herein whether sustained GLP-1 activation is protective against the renal vascular dysfunction and CKD in an obese rat model on a high-salt diet. Therefore, our aims were 1) to directly assess renal endothelial and smooth muscle dysfunction within the renal vascular network of male obese/lean Zucker rats maintained on a high-salt diet using in vivo micro-angiography; 2) to assess the direct impact of sustained GLP-1 activation on renal vascular function, glomerular filtration rate (GFR), albumin creatinine ratio, and blood urea nitrogen (BUN) levels; and 3) to appraise the overall protective effect on GLP-1 agonist treatment on ROS and inflammation within the renal vascular network. Our hypothesis was that LIRA ameliorates the increased renal vasoconstriction and renal dysfunction evoked by a high-salt diet in fa/fa rats directly through increased protein expression of renal endothelial NO synthase (eNOS) and enhanced endothelium-dependent
NO-mediated dilation in renal small arteries, and indirectly by lowering arterial blood pressure relative to vehicletreated rats.

\section{Materials and Methods}

Animals and Group Allocation. All animal experiments were performed in accordance with the National Cerebral and Cardiovascular Centre (Osaka, Japan) animal care committee (approval number: 16086) according to National Institutes of Health guidelines. Thirty-two 8-week-old male Zucker lean $(+/+)$ and obese (fa/fa) rats were purchased from Japan SLC (Hamamatsu, Japan). Zucker $+/+$ rats were divided into two groups to receive either saline [lean (Ln)vehicle (Veh)] or LIRA ( $0.1 \mathrm{mg} / \mathrm{kg}$ per day) (Ln-LIRA), and Zucker fa/fa rats were divided into two groups to receive either saline (fa/fa-Veh) or LIRA ( $0.1 \mathrm{mg} / \mathrm{kg}$ per day) (fa/fa-LIRA) for 8 weeks. Vehicle and LIRA were administered subcutaneously via osmotic mini-pumps (2ML-4; Alzet). The clinically relevant dose of LIRA was selected based on previous studies (Hendarto et al., 2012; Mima et al., 2012; Fujita et al., 2014). All rats were provided with ad libitum access to normal drinking water and a high-salt chow $\operatorname{diet}(6 \% \mathrm{NaCl}$ w/v; Oriental Yeast Co., Ltd., Tokyo, Japan). LIRA (Victoza) was purchased from Japan. The high-salt diet and LIRA treatment were initiated at the same time to determine the protective beneficial effects against highsalt-induced renal dysfunction. At completion of the main study a supplementary angiography experiment was then performed to investigate the renal vascular response to acetylcholine $(\mathrm{ACh})$ and sodium nitroprusside (SNP) in Zucker $+/+$ rats $(n=5)$ on a normalsalt chow $\operatorname{diet}(\mathrm{NaCl} 0.44 \% \mathrm{w} / \mathrm{v})$.

Blood Pressure Measurements. Blood pressure was measured noninvasively in all conscious rats using the tail-cuff method (BP98A-L; Softron Corporation, Tokyo, Japan) in a $36-37^{\circ} \mathrm{C}$ preheated platform for about 10-15 minutes following training sessions prior to recording days. The mean of four to five systolic blood pressure recordings were determined for each rat at the end of the 8-week treatment following recordings at the start and mid-study for accustomization.

Transcutaneous GFR Measurement in Conscious Rats. Fluorescein isothiocyanate/sinistrin half-life was measured prior to high-salt diet feeding and after 8 weeks of vehicle or LIRA treatment on the high-salt diet. Animals were induced with $4 \%$ isoflurane and anesthesia was maintained at $2 \%$ until the completion of device fixation and tail vein injection. For the measurement of GFR, a transcutaneous non-invasive clearance kidney device (Mannheim Pharma \& Diagnostics GmbH, Mannheim, Germany) was fixed to the depilated skin on the back of rats using a double-sided adhesive patch following the procedures described previously (Herrera Pérez et al., 2016). Fluorescein isothiocyanate-sinistrin were dissolved in physiologic saline and administered through tail-vein injection (10 mg/100 g b.wt.; Fresenius Kabi Austria GmbH, Linz, Austria) (Ellery et al., 2015). Animals were allowed to fully recover and move freely within a cage until transcutaneous recordings were stopped after 2 hours and 15 minutes. Fluorescein isothiocyanate/sinistrin excretion half-life was calculated using a one-compartment model (MPD Laboratory version 1.0; Mannheim Pharma \& Diagnostics $\mathrm{GmbH}$ ) conversion equation as previously described (Herrera Pérez et al., 2016).

Measurement of Albumin, Albumin Creatinine Ratio, and BUN. Urinary levels of albumin and albumin-creatinine ratio were measured in a single rapid colorimetric assay format using a Spectrum Diagnostic Kit (DCA 2000 Vantage analyzer, Siemens, Eschborn, Germany) according to the manufacturer's protocol. BUN was measured by colorimetric qualitative analysis using commercially available Fuji Dri Chem slides (DRI-CHEM 7000; Fujifilm, Tokyo, Japan).

Measurement of Nonfasted Insulin and Glucose Levels. Insulin was determined on frozen plasma samples using a rat insulin ELISA kit (AKRIN-010RU; Fujifilm Wako Pure Chemical Corporation) 
according to the manufacturer's recommendations. Blood glucose levels were measured using a FREESTYLE glucometer (Abbott, Chicago, IL).

Surgical Preparation of Rats for Microangiography. Rats were induced with $4 \%$ isoflurane and anesthesia was maintained at $2.5 \%$ isoflurane throughout surgery. After anesthesia was achieved, the trachea was intubated with an angiocath sheath and connected to a small animal ventilator (Model SN-480-7; Shinano Seisakusho Co., Ltd, Tokyo, Japan). Ventilation was volume controlled at a frequency of 60 per minute with a tidal volume $8-10 \mathrm{ml} / \mathrm{kg}$ of body weight. Rectal temperature was monitored and regulated at $36-37^{\circ} \mathrm{C}$ using a combination of heating pad and/or heating lamp throughout the experimental procedure. The right common carotid artery was cannulated with a polyethylene catheter to record mean arterial blood pressure and heart rate via a pressure transducer (MLT 0670; $\mathrm{AD}$ instruments, Castle Hill, Australia). Then, the right jugular vein was cannulated for continuous infusion of vehicle Ringer's lactate solution $(4 \mathrm{ml} / \mathrm{h})$ (Otsuka Pharmaceuticals, Chiyoda, Japan) and drug solutions were used to assess vascular function. The right femoral artery was also cannulated retrogradely, and the tip of the catheter (BD Medical Technology) was positioned just below the level of the left renal artery for contrast medium infusion.

X-Ray Microangiography System and Preparation. Following surgical preparation, the rats were placed in the microfocus X-ray system (MFX-80HK; Hitex, Osaka, Japan) for terminal microangiography experiments under isoflurane anesthesia $(2 \%)$. The X-ray system consisted of an open-type 1- $\mu \mathrm{m}$ microfocus X-ray source (L9191; Hamamatsu Photonics, Hamamatsu, Japan) and a 50/100 mm dual-mode X-ray image intensifier with a chargecoupled device camera (E5877JCD1-2 N; Toshiba, Tokyo, Japan) operating at $80 \mathrm{kV}$ and $40 \mu \mathrm{A}$ current. Microangiography image sequences were recorded digitally at a rate of $30 \mathrm{frames} / \mathrm{s}$, as a series of $640 \times 480$ pixel bitmap image files. Each supine rat was positioned on the sample plate above the X-ray source such that the left kidney was positioned in the center of the X-ray detector field of view. Through an abdominal incision, viscera and fat pads were transected away for an uninterrupted view of the left kidney to avoid superimposition of mesenteric arteries. Vessel internal diameter determination was calibrated by placing a $50-\mu \mathrm{m}$ calibration tungsten wire on the surface of the exposed left kidney, which was kept moist with topical application of glycerol. After verifying that the imaging field covered the left renal arteries and most of the left kidney, heparin-diluted $(100 \mathrm{U} / \mathrm{ml})$ iodinated contrast medium (Iomeron 350; Eisai, Tokyo, Japan) was injected intra-arterially as a bolus (250-300 $\mu \mathrm{l}$ at a speed of $30 \mathrm{ml} / \mathrm{min})$ with a syringe injection pump (PHD-2000; Harvard Apparatus). X-ray image acquisition was initiated from 1 second before iodine injection and continued for 5 seconds. Subtraction angiogram image analysis was performed as previously described (Sonobe at al., 2015). Rats were then overdosed with $4 \%$ isoflurane followed by potassium chloride injection and the kidney was excised for further analysis.

Renal Angiography Protocol and Rationale. Renal angiograms were recorded sequentially at the end of a 5-minute baseline of vehicle infusion $(3.0 \mathrm{ml} / \mathrm{h}$ of Ringer's lactate), ACh $(3.0 \mu \mathrm{g} / \mathrm{kg}$ per minute for 5 minutes), and SNP (3.0 $\mu \mathrm{g} / \mathrm{kg}$ per minute for 5 minutes), followed by combined blockade of prostaglandin production with the cyclooxygenase (COX) inhibitor indomethacin (Indo) $(10 \mathrm{mg} / \mathrm{kg}$ i.v. bolus) and uncoupling of myoendothelial gap junctions with carbenoxolone administration (carbenox, $10 \mathrm{mg} / \mathrm{kg}$ i.v. bolus) and 10 -minute equilibration period. We then assessed endothelium-dependent dilation after blockade with a repeat infusion of ACh $(3.0 \mu \mathrm{g} / \mathrm{kg}$ per minute) (Fig. 5B). Aging and insulin resistance have both been shown to impair conducted dilation, which is the transmission of hyperpolarization along vessels via endothelial cell-to-cell connections and smooth muscle cell-to-cell connections involving various potassium channels and connexins (Feher et al., 2014; Chen et al., 2016). A reduction in $\mathrm{NO}$ bioavailability has been shown to impair conducted dilation in coronary arterioles by reducing NO-mediated facilitation of outward extracellular potassium ion efflux and subsequent smooth muscle hyperpolarization (Feher et al., 2014). Blockade with indomethacin + carbenox was used in this study to inhibit local radial dilation through prostanoids and myoendothelial gap junctions to determine if reduced NO availability due to metabolic syndrome impaired conducted dilation within the renal vasculature, and whether LIRA enhanced conducted dilation. Changes in internal vessel diameter or caliber of the same vessels that were visualized in all treatment periods are considered in this study to be indicative of local (radial) dilatory function. On the other hand, a change in the number of angiographically visualized vessel segments during stimulation (i.e., not seen at baseline) is used here as an index of a change in distal or conducted dilation to the renal cortex. Therefore, we expected that any significant decrease in vessel internal diameter or lack of change in internal diameter during stimulation of the renal vasculature with ACh or SNP would indicate that there is underlying endothelial dysfunction or smooth muscle dysfunction, respectively, involving impairment of NO signaling. On the other hand, a reduction in newly visualized vessels during ACh or SNP stimulation or a lack of change in visualized vessel number would indicate that there is impairment in at least one of the mechanisms responsible for conduction of distal dilation and regulation of global perfusion [NO and endothelium-derived hyperpolarizing factor (EDHF)]. Conversely, an increase in newly visualized vessels during ACh stimulation in LIRA-treated rats would indicate that the distal increase in perfusion is facilitated by enhanced release of NO and/or EDHF induced by LIRA treatment.

Measurement of Internal Vessel Diameter. Image analysis was performed as previously described using Image J (version 1.48; National Institutes of Health) (Sonobe et al., 2015). Quantitative analysis of vessel internal diameter was based on measurements from the middle of the discrete vessel segments in the subtracted average images obtained from individual rats during each treatment period.

Histologic Examination of the Kidney. Following excision, two-thirds of the right kidney samples was immersed in $4 \%$ paraformaldehyde for more than 48 hours and then embedded either in paraffin or an optimum cutting temperature compound (Tissue Tek, Sakura Finetek Japan Co., Ltd., Tokyo), whereas the remaining onethird was frozen in liquid nitrogen for western blotting and stored at $-80^{\circ} \mathrm{C}$ until assays were performed. Paraffin-embedded kidney sections were stained with Azan-Mallory stain. For evaluation of collagen fiber content (staining blue), images were taken randomly under light microscopy $(\times 400)$ from 20 different microscopic fields per section using a BX53 microscope (Olympus, Tokyo, Japan) equipped with a complementary metal-oxide-semiconductor camera (DP-80; Olympus).

Immunofluorescence. Frozen sections were dried at room temperature for 10 minutes and rinsed three times in PBS for 5 minutes. Sections were blocked for 1 hour in blocking buffer (1\% bovine serum albumin and $0.1 \%$ Triton $\times 100$ diluted in PBS). Sections were then incubated with anti-mouse CD-68 (1:500; ab-31630) overnight at $4^{\circ} \mathrm{C}$. The slides were washed three times for 5 minute in PBS and incubated for 1 hour at room temperature with the conjugated secondary antibody (11004A, goat anti-mouse Alexa Fluor 568, 1/400; Life Technologies).

Protein Expression Determination by Western Blotting. Right kidney tissue samples were homogenized with lysis buffer. Renal protein concentrations in these homogenized samples were measured by using the bicinchoninic acid assay method. For western blots, proteins were separated by SDS-PAGE and the following antibodies were used to quantify the renal protein expression levels: anti-vascular endothelial growth factor [(VEGF), 1:1000; sc-152); antiTGF- $\beta 1$ (1:1000; sc-146) and anti-COX-2 (1:1000, NB100-689; Novus Biologicals) rabbit polyclonal antibodies; and anti- $\beta$-actin (1:1000, sc-47778), anti-eNOS (1:1000, sc-376751), anti-nitrotyrosine [(NT); 1:1000, sc-32757], and anti-GLP-1R (1:1000, sc-390773) mouse polyclonal antibodies (Santa Cruz Biotechnology). For SDS-PAGE, $50 \mu \mathrm{g}$ of protein was loaded on $10 \%$ sodium dodecyl 
sulfate-polyacrylamide gels (Bio-Rad), and subsequently the proteins were transferred to nitrocellulose membranes. These membranes were then blocked with Blocking One solution (Nacalai Tesque Inc., Kyoto, Japan) or 3\% bovine serum albumin (Sigma, St. Louis, MO) in Tris-buffered saline/Tween 20 (20 mM Tris, pH 7.6, $137 \mathrm{mM} \mathrm{NaCl}$, and $0.05 \%$ Tween 20) at room temperature. After incubation with the primary antibody, the bound antibody was visualized with the respective horseradish peroxidase-coupled secondary antibody (1:10000; Cell Signaling Technology, Inc.). Chemiluminescence signals were visualized using a chemiluminescence imaging system (Ez-Capture-II; ATTO Corporation, Tokyo, Japan) and quantified using $\beta$-actin signal as a protein loading control.

Gene Expression Analysis. RNA was extracted from kidney tissues using TRIzol lysis reagent in conjunction with the RNA Purification kit (RNeasy Mini Kit; QIAGEN) with on-column DNase treatment (RNase-Free DNase Set; QIAGEN). The quantity of purified RNA was measured by using the Nano Drop One Analyzer (Thermo Fisher Scientific) and the RNA quality from each sample was assured by the A260/280 absorption ratio. Then, $2 \mu \mathrm{g}$ of total RNA was reverse transcribed using the Complimentary DNA Synthesis Kit (Superscript VILO; Invitrogen, Thermo Fisher Scientific) as per the manufacturer's instructions. Real-time polymerase chain reactions were run on a StepOne 7500 Real-Time PCR System (Applied Biosystems, Foster City, CA) using Taqman Universal Fast PCR Master Mix and gene-specific predesigned Taqman primers obtained from Applied Biosystems [GenBank accession numbers: Rn02132634_s1 (eNOS); Rn00561129_m1 [endothelin-1 (ET-1)]; Rn00569575_m1 [fibronectin (FN-1)]; Rn01775763_g1 (GAPDH); Rn00577994_g1 (glutathione peroxidase-1); Rn00586652_m1 (NADPH oxidase, Nox-1); Rn00572010_m1 (TGF- $\beta 1$ ); Rn00563627_m1 (VCAM-1); and Rn01511602_m1 (VEGF)] (Applied Biosystems). Each sample was run in duplicate, and the comparative threshold cycle $(\Delta \Delta \mathrm{CT})$ method was used to analyze the genes of interest relative to the GAPDH reference gene as previously described (Livak and Schmittgen, 2001).

Statistical Analysis. Data are presented as mean \pm S.E.M. The mean baseline and percentage change in vessel internal diameter and visible vessel number from baseline of each branching order in individual rats (within-animal average) were pooled for group comparisons. To compare the mean values of variables among the rat treatment groups we used either one-way ANOVA or two-way repeated measures ANOVA to compare time-dependent changes between groups, such as GFR, urine albumin, and urine albumin creatinine ratio over time. $P<0.05$ was considered statistically significant. Furthermore, parameters obtained from the four-rat groups were subjected to two-way ANOVA to evaluate significance of differences due to either genotype (fa/fa vs. +/+) or drug treatment (LIRA vs. vehicle), or their interaction (interaction and genotype $\times$ treatment). Prism version 5.0 software by GraphPad Software (La Jolla, CA) was used for statistical analysis.

\section{Results}

LIRA Treatment Had No Impact on Body Weight or Blood Glucose but Improved Plasma Insulin Levels. The body weight of rats was comparable within $\mathrm{Ln}$ and fa/fa groups throughout the duration of the study, with no significant differences observed between LIRA and saline-treated groups (Fig. 1A). Blood glucose levels were not significantly altered by long-term administration of LIRA, with no significant treatment effect observed (Fig. 1B). However, salinetreated fa/fa rats showed greatly elevated plasma insulin levels when compared with Ln group rats, but LIRA treatment tended to decrease insulin levels in fa/fa rats (treatment; $P=0.089$ ) (Fig. 1C).

LIRA Suppressed Blood Pressure Elevation in Conscious fa/fa Rats. Figure 1D demonstrates that 8 weeks after high-salt diet there was a trend toward systolic blood pressure to be mildly elevated in fa/fa-Veh group rats compared with Ln-Veh rats $(133.4 \pm 5.2$ vs. $126.1 \pm 3.8 \mathrm{~mm}$ $\mathrm{Hg} ; P=0.063)$. In contrast, LIRA-treated fa/fa rats had significantly lower systolic blood pressure compared with those of fa/fa-Veh group rats $(122.2 \pm 3.5$ vs. $133.4 \pm$ $5.2 \mathrm{~mm} \mathrm{Hg} ; P=0.013$ ) (Fig. 1D).

LIRA Effect on GFR. GFR was higher during the baseline $(0$ week) in fa/fa rats compared with $\mathrm{Ln}$ rats (genotype; $P=0.001$ ). After 8 weeks on high-salt diet, GFR in fa/fa-Veh group rats was significantly decreased in comparison with the Ln-Veh rats $(0.82 \pm 0.05$ vs. $1.50 \pm 0.05 \mathrm{ml} / \mathrm{min} ; P<0.01)$ (Fig. 1E). LIRA-treated fa/fa rats maintained near normal GFR in contrast to the vehicle-treated fa/fa rats $(1.33 \pm 0.06$ vs. $0.82 \pm 0.05 \mathrm{ml} / \mathrm{min} ; P<0.001$ ).

LIRA Partially Reduced BUN and Albuminuria in fa/fa Rats. Urinary albumin and albumin-creatinine ratio were elevated significantly in fa/fa-Veh group rats compared with Ln-Veh rats (Fig. 1, G and H). Eight weeks of LIRA treatment in the fa/fa rats resulted in decreased BUN levels relative to fa/fa-Veh rats $(P=0.032)$ (Fig. $1 \mathrm{~F})$. Furthermore, LIRA treatment partially decreased urinary albumin $(49.9 \pm 7.6$ vs. $74.5 \pm 4.7 \mathrm{mg} / \mathrm{l} ; P<0.01$ ) (Fig. $1 \mathrm{G}$ ) and albumin/creatinine ratio (254.9 \pm 54.6 vs. $371.9 \pm 24.7 \mathrm{mg} / \mathrm{l} ; P<0.001)$ (Fig. $1 \mathrm{H})$.

LIRA Did Not Alter the Profibrotic and Inflammatory Gene Expression Profile. Renal gene expression analyses revealed that the obese phenotype was associated with a significantly higher expression of ET-1, NOX1, VCAM- 1 , and TGF- $\beta 1$ mRNA on a high-salt diet (Fig. 2, A-D). However, mRNA expression of the antioxidative pathway glutathione peroxidase-1, FN-1, and genes important for vascular homeostasis (eNOS and VEGF) was not different between fa/fa and Ln rats (Fig. 2, E-H). LIRA did not alter gene expression for any gene examined, with the notable exception of VCAM-1, which showed a strong trend to be reduced by LIRA (treatment; $P=0.061)$.

LIRA Treatment Improved eNOS and VEGF Expression without Appreciably Changing Markers of Nitrosative Stress, Prostanoids, or TGF- 11 Levels. Renal protein expression of NT and VEGF did not differ significantly between vehicle-treated fa/fa and $\mathrm{Ln}$ rats (Fig. 3, A, $\mathrm{B}$, and $\mathrm{F}$ ). The obese phenotype was associated with a pronounced increase in macrophage marker protein CD-68 (genotype; $P=0.007$ ) (Fig. $3 \mathrm{C}$ ) and COX-2 protein (genotype; $P=0.0001$ ) (Fig. $3 \mathrm{G}$ ), while there were similar trends for increased TGF- $\beta 1$ (Fig. 3D) and eNOS (Fig. 3E) (genotype; $P=0.052$ ) protein. Treatment with LIRA did not significantly alter NT, CD-68, TGF- $\beta 1$, or COX-2 protein levels (Fig. 3, B-D and G), but did restore expression of VEGF (treatment; $P=0.063$ ) (Fig. 3 F) and nearly doubled mean eNOS expression (treatment; $P=0.014$ ) (Fig. $3 \mathrm{E}$ ) in fa/fa LIRA-treated rats compared with fa/fa-Veh and Ln rats.

LIRA Treatment Attenuated GLP-1R Expression. GLP-1R protein expression in the kidney was increased in $\mathrm{fa} / \mathrm{fa}$ rats relative to $\mathrm{Ln}$ rats. Pronounced variability in protein expression levels was observed in LIRA-treated fa/fa rats 
A

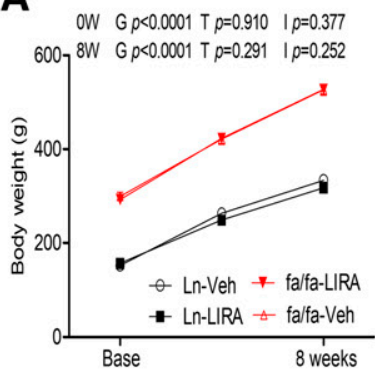

E ow $G p<0.0001 T p=0.265 \quad p=0.084$

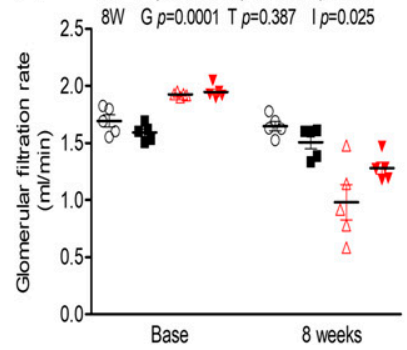

B

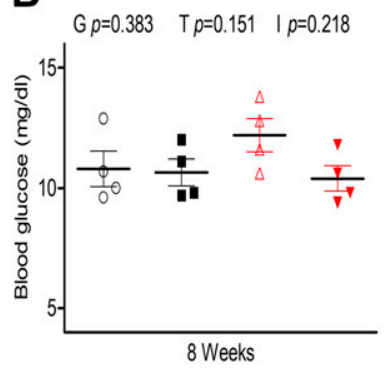

$\mathbf{F}$

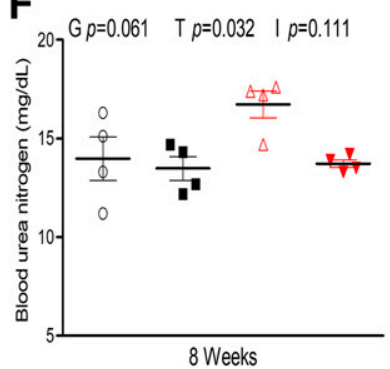

C

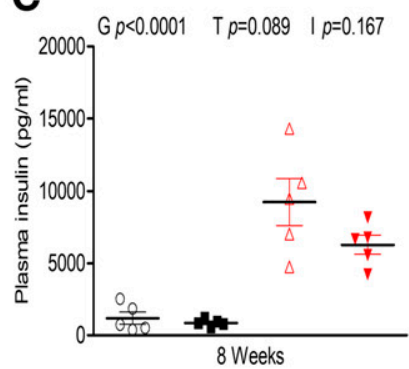

D

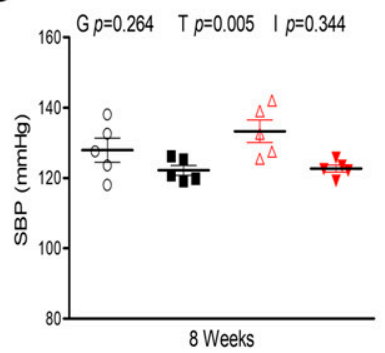

G

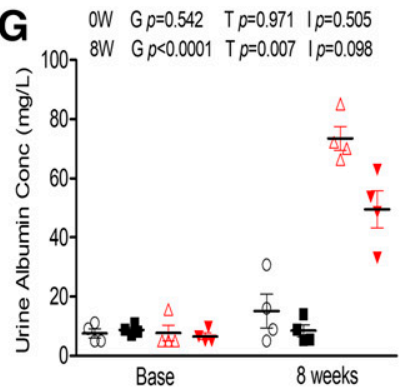

H OW $G p<0.0001 T p=0.475 \quad \mid p=0.735$ 5007 W $\quad$ G $p=0.0001$ T $p=0.032 \quad \mid p=0.097$

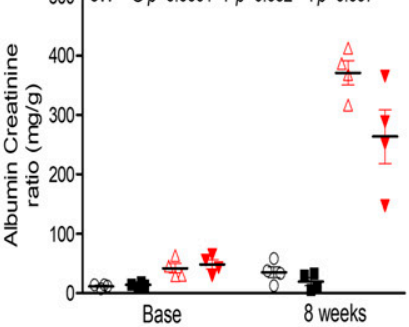

Fig. 1. Effects of LIRA on (A) body weight, (B) blood glucose, (C) plasma insulin levels, (D) systolic blood pressure (SBP), and (F) blood urea nitrogen levels in Zucker rats after 8 weeks on a high-salt diet. The effects of LIRA treatment on glomerular filtration rate (E), urinary albumin $(\mathrm{G})$, and albumin creatinine ratio $(\mathrm{H})$ in Zucker rats during baseline and after 8 weeks on a high-salt diet. The data are shown as the mean \pm S.E.M.; $N=4-7$ rats per group. The significance of group differences was determined by two-way ANOVA for factors of genotype (G) and drug treatment $(\mathrm{T})$ and their interaction (I).

compared with vehicle-treated rats, but LIRA significantly reduced receptor protein expression (treatment; $P=0.046$ ) (Fig. 3, A and H). However, immunoreactive GLP-1R expression was not detected by immunofluorescence in the Zucker rat kidney (data not shown), even though the antibody was sufficiently sensitive to enable detection of endogenous
GLP-1R protein expression in kidney tissue protein extract (Fig. 3, A and $\mathrm{H}$ ).

LIRA Reduced Tubulointerstitial Fibrosis in fa/fa Rats. Collagen accumulation was evaluated to assess the extent of fibrosis. In the kidneys of the rats from both Ln groups, collagen deposition (Fig. 4A, blue color)
A

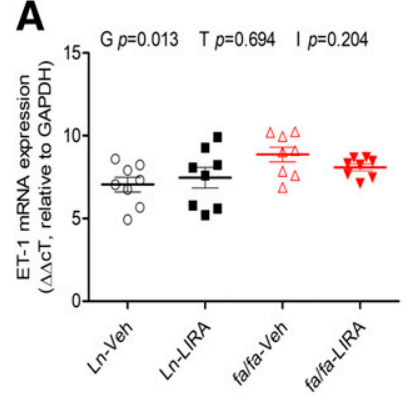

E

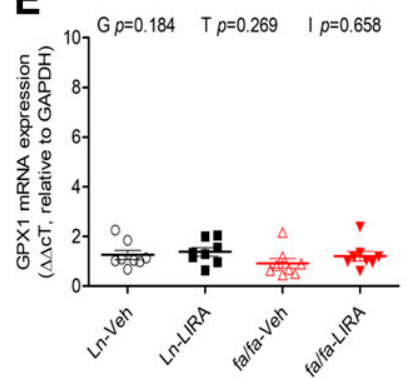

B

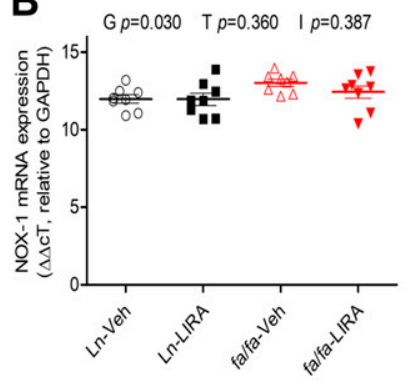

$\mathbf{F}$

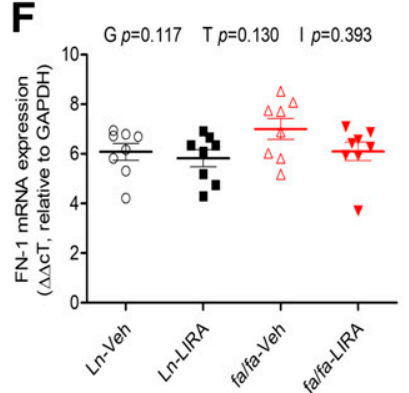

C

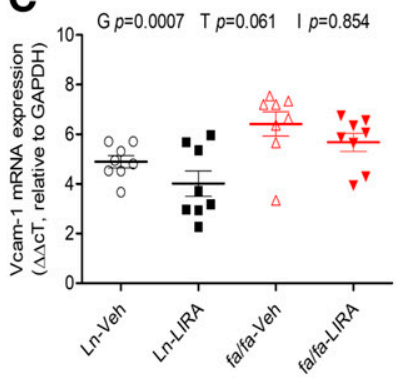

G

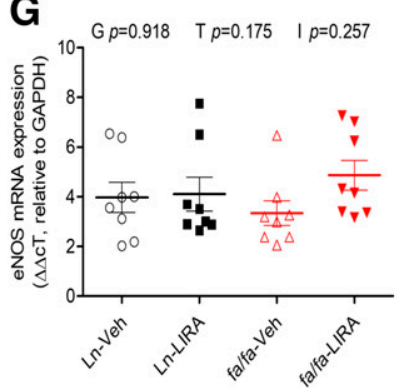

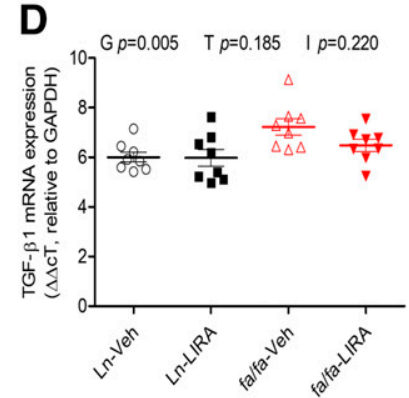

H

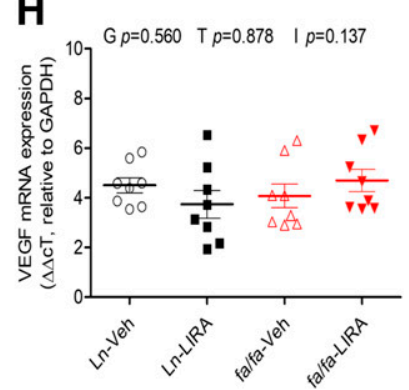

Fig. 2. The effects of LIRA treatment on renal mRNA expression of ET-1, NADPH oxidase 1 (Nox-1), VCAM-1, TGF- $\beta 1$, glutathione peroxidase-1 (GPX-1), FN-1, eNOS, and VEGF (A-H). GAPDH gene $\Delta \Delta$ CT value was used as an internal control. The data are shown as the mean \pm S.E.M.; $N=8$ rats per group. The significance of group differences was determined by two-way ANOVA for factors of genotype $(\mathrm{G})$ and drug treatment (T) and their interaction (I). 
A
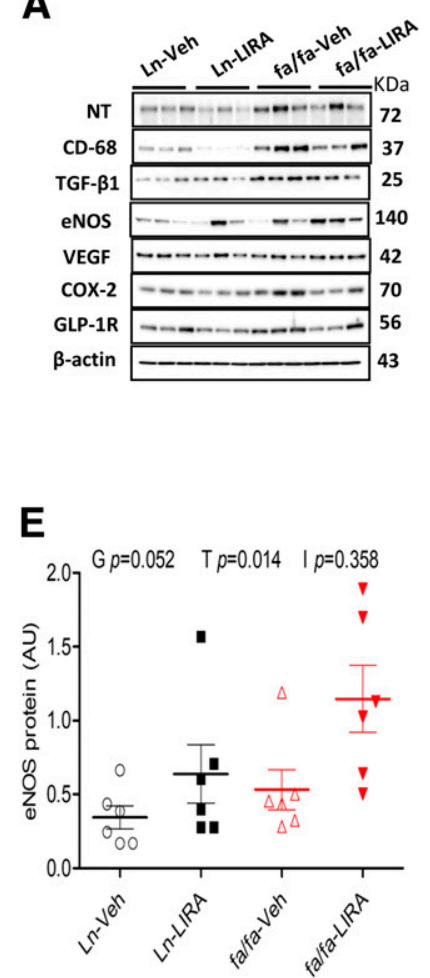

B

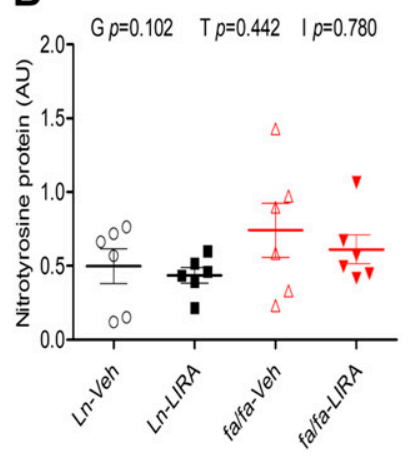

F

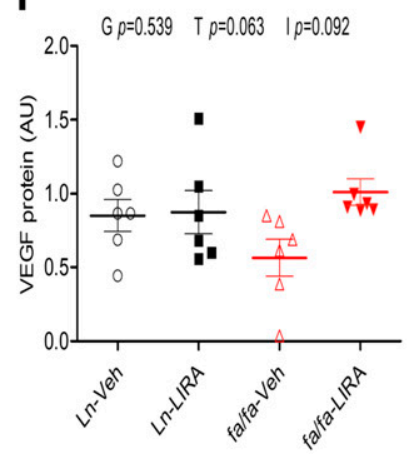

C

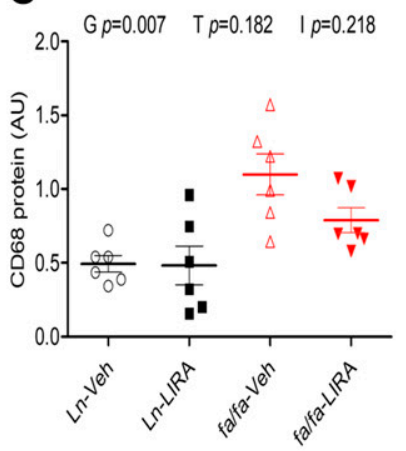

G

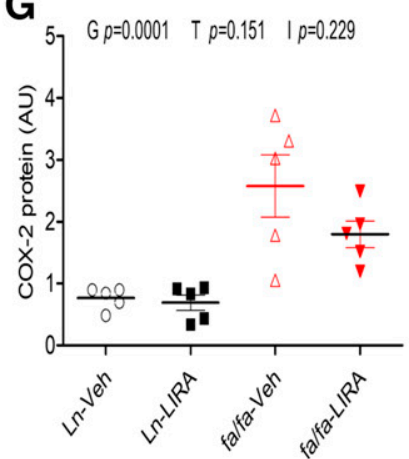

D

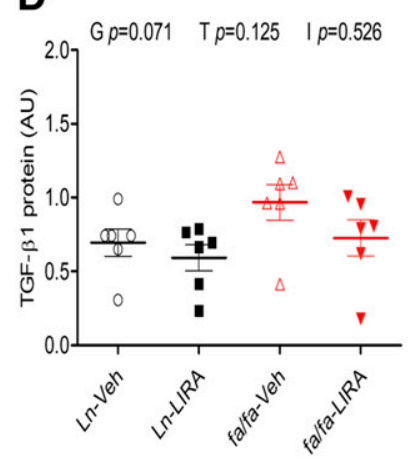

H

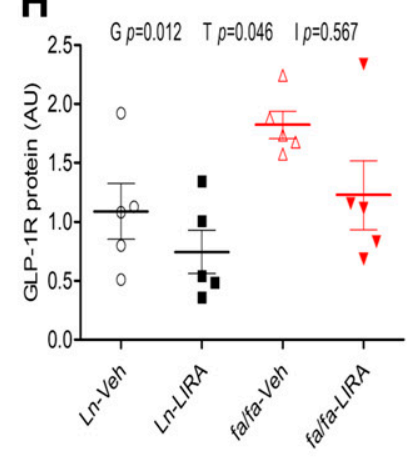

Fig. 3. The effects of LIRA treatment on protein expression of NT, CD-68, TGF- $\beta 1$, eNOS, VEGF, COX-2, and GLP-1R in the kidney. (A) Protein expression levels of NT, CD-68, TGF- $\beta 1$, eNOS, VEGF, COX-2, and GLP-1R detected by western blotting. $\beta$-Actin was used as an internal control. Bands are representative of seven separate experiments. (B-H) Relative density values of NT, CD-68, TGF- $\beta 1$, eNOS, VEGF, COX-2, and GLP-1R. The data are shown as the mean \pm S.E.M.; $N=5$ to 6 rats per group. The significance of group differences was determined by two-way ANOVA for factors of genotype (G) and drug treatment (T) and their interaction (I).

was observed in small amounts including around the glomeruli, vessels, and borders of the tubules. Compared with Ln rats, collagen accumulation was significantly increased in $\mathrm{fa} / \mathrm{fa}-\mathrm{Veh}$ rats (Fig. 4B), even though the levels of TGF- $\beta 1$ protein upregulation did not reach statistical significance (Fig. 3, A and D). In contrast, collagen accumulation was partially attenuated by LIRA treatment (Fig. 4, A and C).

LIRA Suppressed Renal Macrophage Infiltration and Glomerular Enlargement. Figure $4 \mathrm{~B}$ presents representative immunofluorescence images of macrophages within kidneys. In fa/fa-Veh group rats CD-68 positive cells were observed significantly more often within the glomeruli compared with Ln rats. Macrophage accumulation was attenuated by LIRA treatment $(P<0.001)$ (Fig. $4, \mathrm{~B}$ and D). However, western blotting of renal tissue did not reveal a statically significant reduction in CD-68 marker protein (Fig. 3, A and C). Although all rats were on a high-salt diet, the glomeruli in fa/fa-Veh rats were $~ 40 \%$ larger than in Ln rats (by crosssectional area and perimeter). LIRA treatment ameliorated the expansion of glomerular volume compared with fa/fa-Veh rats (Fig. 4, E and F).

Baseline Hemodynamics. Mean arterial pressure (MAP) during baseline was slightly elevated in vehicle-treated fa/fa rats compared with $L n$ rats $(118.7 \pm 9.8$ vs. $102.3 \pm 6.2 \mathrm{~mm}$ $\mathrm{Hg}$ ), while mean heart rate was comparable (323.6 \pm 20.8 vs. $350.9 \pm 11.7$ beats per minute) (Fig. 6A). LIRA treatment did not alter MAP at baseline in fa/fa rats $(122.9 \pm 9.6$ vs. $118.7 \pm 9.8 \mathrm{~mm} \mathrm{Hg}$ ), but increased mean heart rate (362.1 \pm 12.1 vs. $323.6 \pm 20.7$ beats per minute) compared with vehicle-treated fa/fa rats (Fig. $6 \mathrm{~A}$ ).
Vessel Internal Diameters and Visualized Vessel Number at Baseline. Representative X-ray angiograms of the renal vasculature are shown in Fig. 5A. Vessel internal diameter in Ln-Veh rats was comparable to Ln-LIRA rats across first-, second-, and third-order arterial vessels. Vehicle-treated fa/fa rat vessel internal diameter was similar to LIRA-treated fa/fa rat vessel internal diameter across first- to third-order branches of arterial vessels (Fig. 5C). Mean total visible vessel branching segments per animal during all treatment periods in the field of view were 10-12 vessels in Ln rats compared with 6-8 in fa/fa-Veh group rats and 9-12 in LIRA-treated fa/fa rats. More third-order vessel branching segments were observed in LIRA-treated fa/fa rats at baseline than vehicle-treated fa/fa rats (Fig. 5, A and D).

LIRA Treatment Improved Renal Dilation to Endothelium-Dependent and -Independent Stimulations. Figure 7A shows the mean change in vessel caliber across first- to third-order branching during ACh infusion. Caliber increases in fa/fa-Veh rats were smaller than Ln rats across second- and third-order vessels. LIRA treatment significantly increased dilation in third-order vessels and there was a similar trend in second-order vessels, but there were no significant differences in the large arteries of firstorder branching. Furthermore, there was a tendency for the number of visible second-order vessels to increase with ACh stimulation $(P=0.07)$ (Fig. $7 \mathrm{~A})$. There was a trend toward a larger decrease in MAP in fa/fa rats in response to ACh compared with $\mathrm{Ln}$ rats $(-12.8 \pm 7.6$ vs. $-1.1 \pm 3.3 \mathrm{~mm}$ $\mathrm{Hg}$, genotype $P=0.078$, Fig. 6B). However, LIRA-treated rats did not differ significantly in the size of the MAP change 

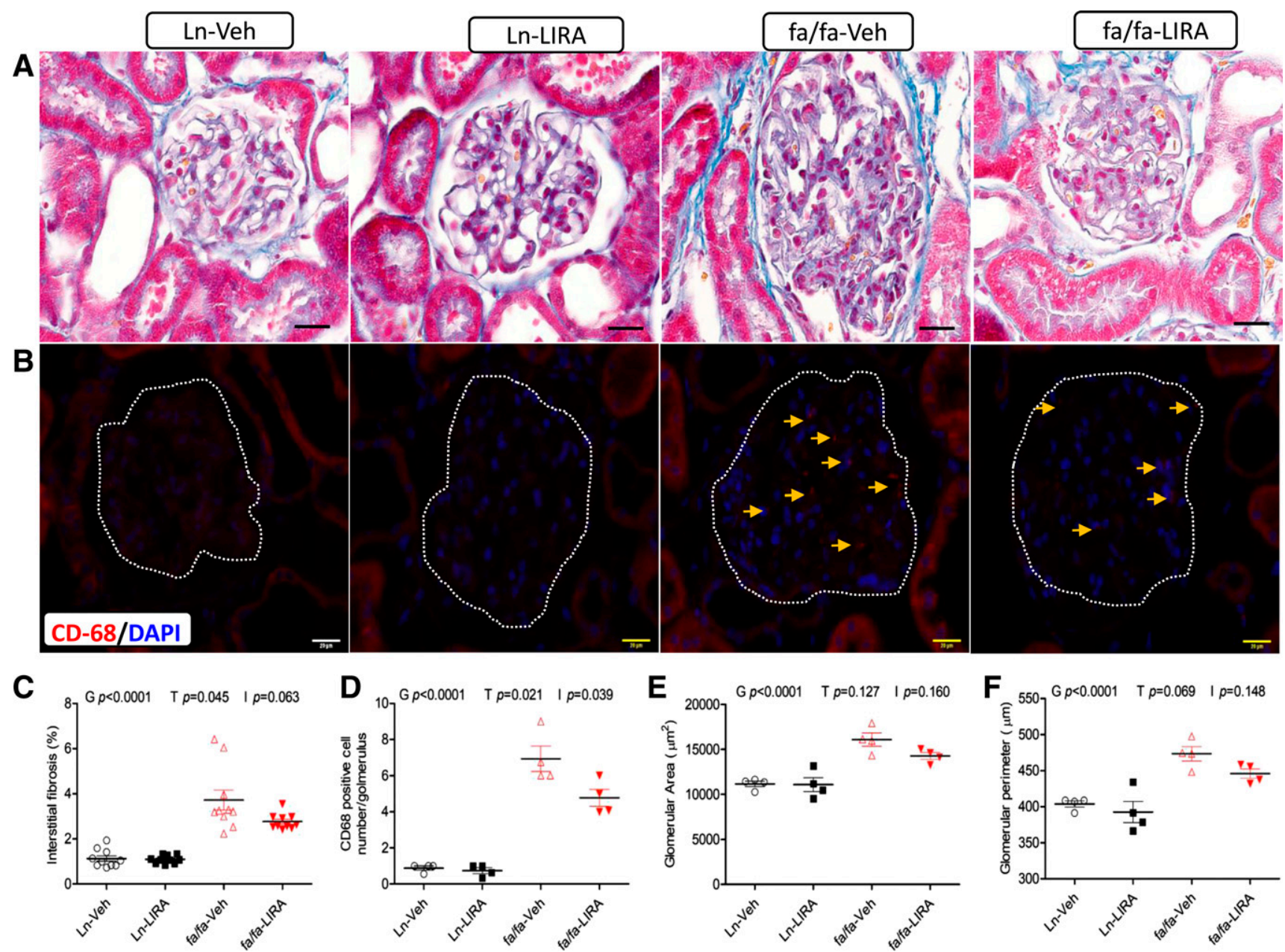

Fig. 4. Effects of LIRA on collagen accumulation and macrophage number in Zucker rat kidneys. (A) Azan-Mallory staining of collagen. AzanMallory-stained kidney sections from Ln-Veh and Ln-LIRA rats showing low levels of collagen deposition (blue fibrils); large patches of strong blue fibrotic tissue reactivity in vehicle-treated fa/fa rats (blue fibrils); LIRA-treated fa/fa rats showing moderate blue fibrous tissue reactivity around glomerulus. Scale bar, $20 \mu \mathrm{m}$. (B) Immunolocalization of macrophage-specific antigen CD-68 in the glomeruli. The merged CD-68/4',6-diamidino-2phenylindole (DAPI) image is presented. (C) Quantification of tubulointerstitial fibrosis (\%). (D) Quantitative measurement of CD-68 positive cell numbers/20 fields; original magnification, $400 \times$. (E and F) Bar diagram shows the mean glomerular area and perimeter $(N=4)$. The significance of group differences was determined by two-way ANOVA for factors of genotype (G) and drug treatment (T) and their interaction (I).

from vehicle-treated rats. Hence, the larger dilator response to ACh in LIRA-treated rats was not due to differences in arterial pressure. Ln-Veh group rats essentially showed no response to SNP across any branching order, and fa/fa-Veh rats showed slight constriction in the smallest vessels (Fig. 7B). On the other hand, SNP evoked potent dilation in LIRA-treated rats that was greatest in the third-order vessels in Ln rats along with a small increase in visible third-order vessels. MAP changes during SNP infusion were similar across all groups, suggesting that the potent dilation only in LIRA rat groups was not due to differences in arterial pressure (Fig. 6B). However, there was nonsignificant modest depression of mean heart rate during SNP treatment in the fa/fa-LIRA rats. Overall, compared with the vehicletreated rats, the LIRA-treated rats exhibited significant enhancement of the vasodilator responses within the renal vasculature that was not directly related to arterial pressure changes at the time of imaging.

In light of the absence of caliber changes to SNP treatment in Ln-Veh rats, we investigated whether this was due to strain influences or the effect of the high-salt diet in $+/+$ rats on a normal chow diet. We found that naive $+/+$ rats had slightly smaller calibers, but not significantly so, in both second- and third-order vessels compared with the Ln-Veh rats (Fig. 8B). In general, renal vessels in the naive $+/+$ rats showed a $20 \%-$ $30 \%$ increase in caliber with a significant increase in the number of perfused third-order vessels during ACh stimulation (Fig. 8D), whereas caliber change during SNP stimulation was $\sim 10 \%$ in the renal vessels (Fig. $8 \mathrm{E}$ ). The latter finding suggests that the sensitivity to NO donors in the renal vascular bed is low in naive Zucker rats and mildly exacerbated by exposure to a high-salt diet (main study).

LIRA Treatment Enhanced Residual Local Dilation Following Blockade of Prostaglandins and Myoendothelial Gap Junctions. MAP decreased significantly in fa/fa rats during blockade, while in $\mathrm{Ln}-\mathrm{Veh}$ rats MAP increased by $\sim 18 \mathrm{~mm} \mathrm{Hg}$ (genotype $P=0.011$ ). Vessel caliber of vehicletreated fa/fa rats showed no change from baseline in first- and second-order vessels, but had a tendency to be constricted in third-order vessels following blockade (Fig. 7C). In contrast, LIRA treatment resulted in a significantly larger caliber in third-order vessels, $\sim 20 \%$ greater than at baseline $(P=0.023)$, 


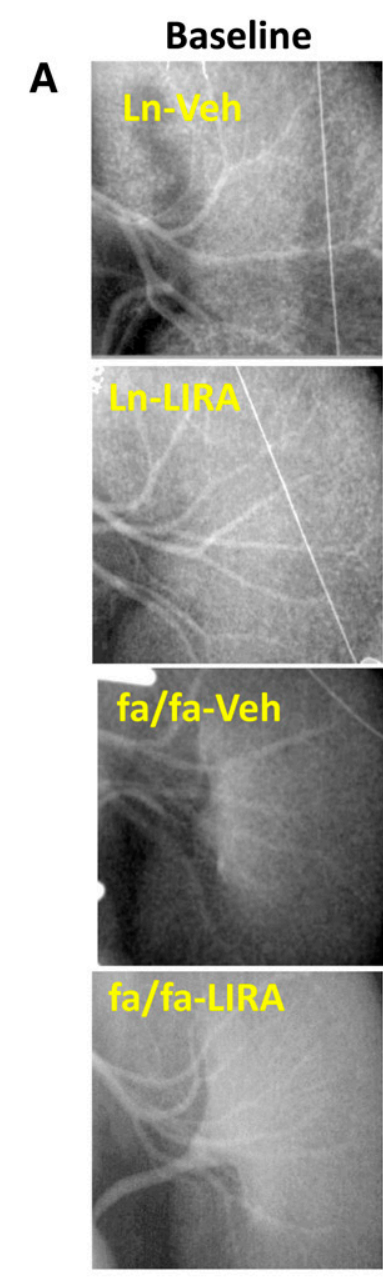

B

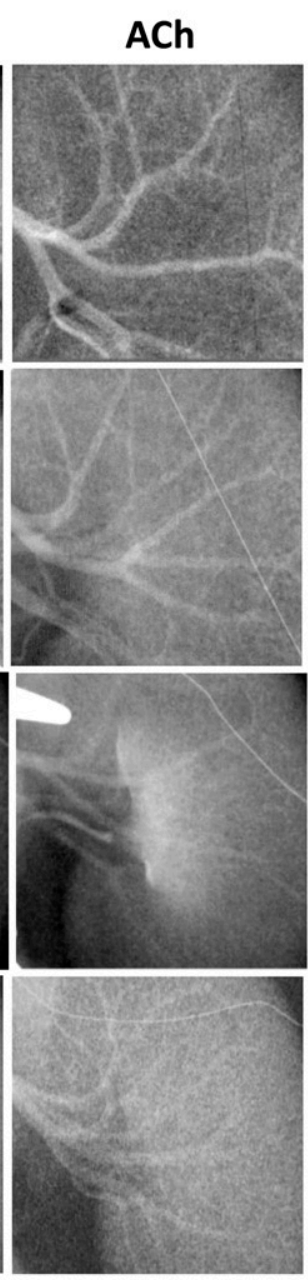

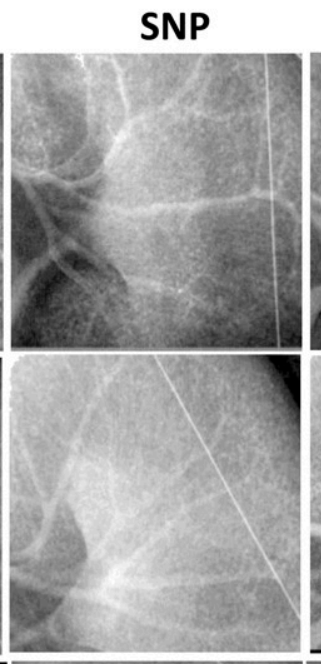
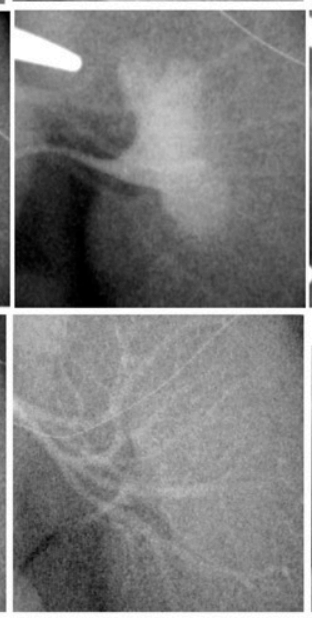

Indo +

Carbenox
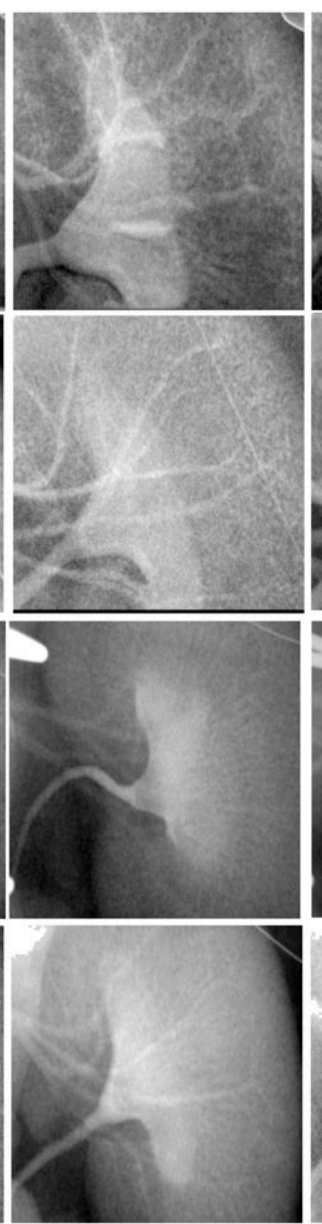

Gap junction blockade

Cyclooxygenase blockade
Indo + Carbenox

$+\mathrm{ACh}$
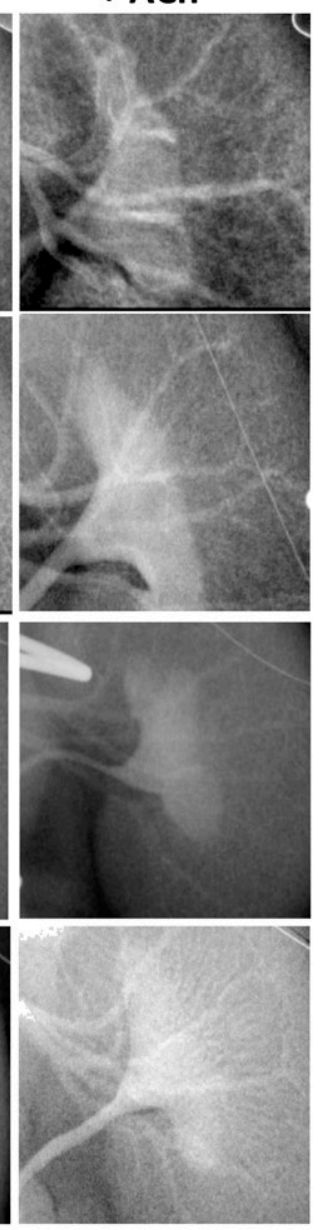

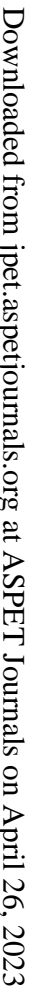
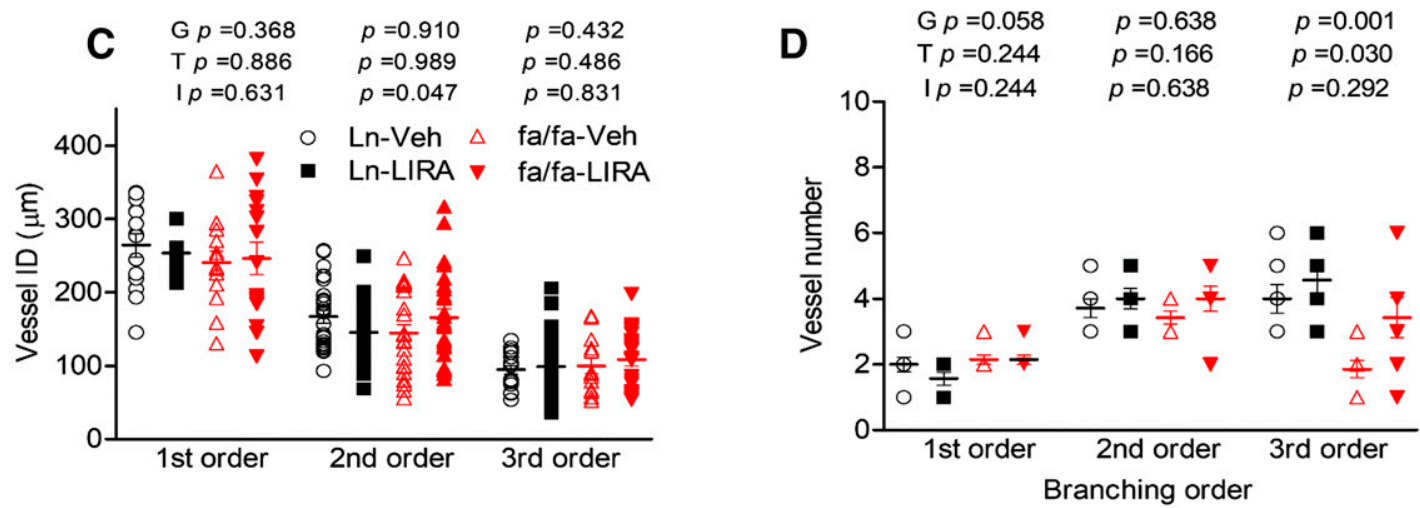

Fig. 5. Representative X-ray angiograms of the renal vasculature in Zucker rats after 8 weeks on a high-salt diet. (A) Images during baseline, infusions of ACh, sodium nitroprusside (SNP), and ACh stimulation during blockade of prostaglandin production and uncoupling of gap junctions. (B) Acute drug infusion protocol during renal vasculature imaging. (C) Vessel internal diameter categorized by branching order during baseline Ringer's lactate infusion. (D) Visualized vessel number during baseline. Calibration wire (white line) $=50 \mu \mathrm{m}$. The significance of group differences was determined by two-way ANOVA for factors of genotype $(\mathrm{G})$ and drug treatment $(\mathrm{T})$ and their interaction (I). 

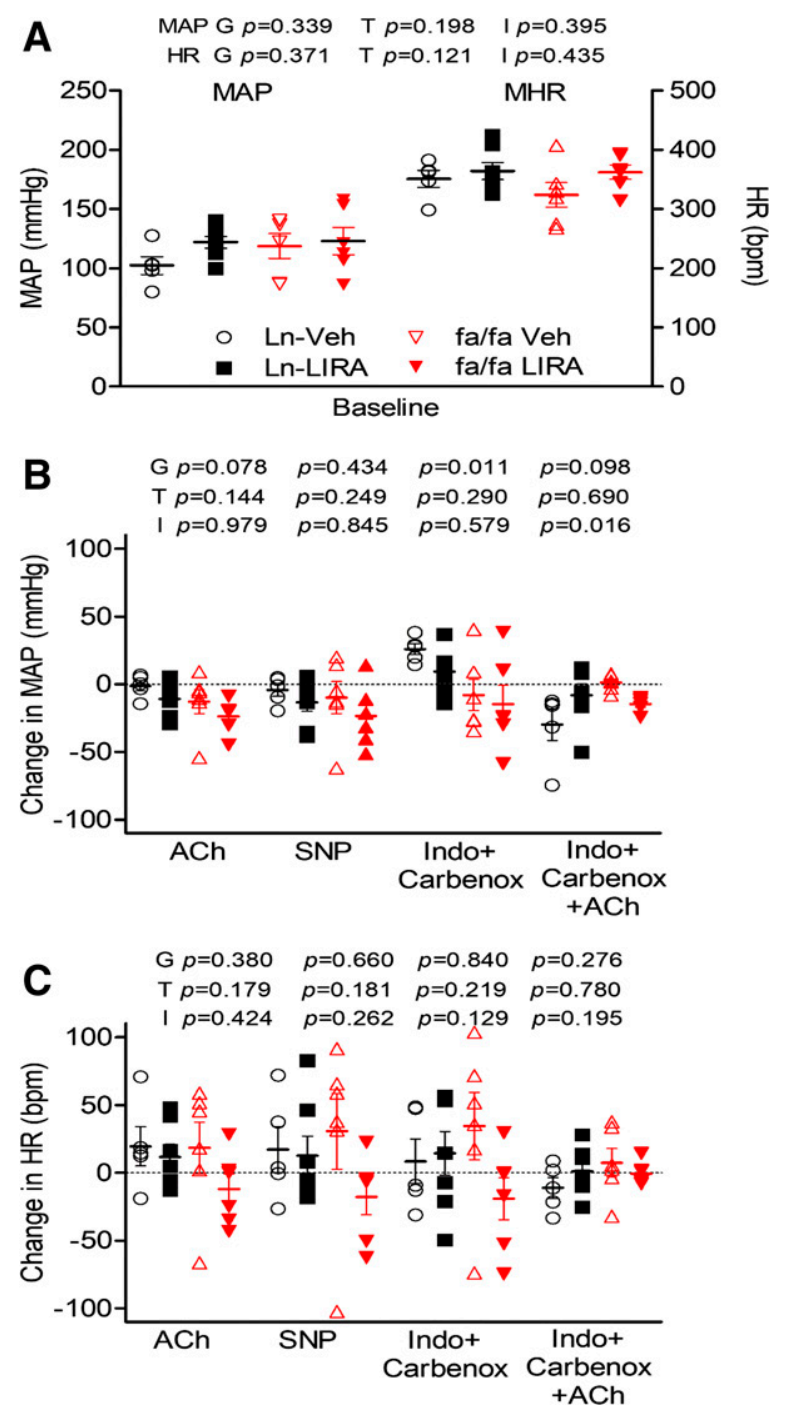

Fig. 6. $M A P$ and mean heart rate (MHR) and their change during the acute infusion. MAP and MHR during baseline $(\mathrm{A})$ and infusions $(\mathrm{B}$ and $\mathrm{C})$ of ACh, SNP, indomethacin + carbenoxolone (blockade), and postblockade + ACh relative to vehicle Ringer's lactate solution (baseline). Mean \pm S.E.M.; $N=5$ to 6 rats per group. The significance of group differences was determined by two-way ANOVA for factors of genotype $(\mathrm{G})$ and drug treatment $(\mathrm{T})$ and their interaction (I).

which did not differ between genotypes. Furthermore, LIRAtreated rats maintained similar visible vessel numbers to baseline in second- and third-order branching during blockade, while vehicle-treated rats showed reduction in secondorder vessels $(P=0.089)$. These findings suggest that dilator prostaglandins most likely contribute to the maintenance of basal renal perfusion in small-to-medium-sized arteries in the vehicle-treated rats irrespective of obesity. However, the small caliber constriction in third-order vessels in fa/fa-Veh rats is likely to be in part due to the small arterial pressure reduction during blockade (Fig. $6 \mathrm{C}$ ). ACh stimulation during blockade of COX and uncoupling of myoendothelial gap junctions evoked a similar degree of increase in vessel internal diameter as that seen during ACh stimulation prior to blockade (Fig. 7D), with only a moderate decrease in MAP observed in Ln rats (interaction; $P=0.016$ ) (Fig. 6B). While local radial dilation was observed in all rats during the final ACh stimulation, we observed that there was a trend toward greater dilation of large first-order arteries in LIRA-treated rats; moreover, there was significantly larger dilation in thirdorder resistance vessels in LIRA-treated rats. There was only a weak trend toward an increase in the number of visible second-order arteries during ACh stimulation post blockade in LIRA-treated rats. Thus, these findings taken together suggest that LIRA enhanced the low sensitivity of renal smooth muscle that we observed in all vehicle-treated rats on a high-salt diet and significantly enhanced endotheliumdependent dilation to ACh to a similar extent in Ln and fa/fa rats, but NO-mediated dilation was generally greater in the $\mathrm{Ln}$ rats. On the other hand, there was little evidence that LIRA modified the extent of conducted dilation within the kidney of Zucker rats.

\section{Discussion}

In the present study, we investigated whether chronic LIRA treatment is able to improve renal vascular function in a rat model of metabolic syndrome and hypertension in part through enhancement of endothelium-dependent dilation in small intrarenal resistance arteries and arterioles. It is well established that the increased risk for cardiovascular disease in prediabetes is multifactorial, with etiologies including insulin resistance, dyslipidemia, hypertension, systemic inflammation, and oxidative stress, as well as renal dysfunction. Recently, it was shown that diabetic nephropathy is improved following treatment with LIRA (Mann et al., 2017). Herein, we showed that low-dose LIRA treatment, first and foremost, maintained NO-mediated dilation in the kidney independent of blood pressure changes and normalized blood pressure in conscious rats to partially restore GFR in the obese Zucker rat model. While blood pressure reduction in conscious rats is likely to contribute to this improvement in renal filtration, the microvessel dilation observed in LIRA rats during ACh and SNP stimulations is not attributable to this blood pressure lowering effect, since basal MAP did not differ between rat treatment groups during anesthesia and the minor nonsignificant MAP changes during stimulations were similar in vehicle-treated rats. Furthermore, LIRA treatment partially prevented glomerular macrophage infiltration, resulting in significant reduction in the development of tubulointerstitial fibrosis but only a modest inhibitory effect on glomerular expansion. Taken together, these findings suggest that LIRA treatment ameliorated the progression of CKD in this study, in part by lowering blood pressure and thereby increasing the capacity for dilation of small intrarenal arteries and arterioles rather than by altering renal oxidative stress per se.

It has already been established that in Zucker fa/fa rats monocyte-macrophage infiltration, glomerular hypertrophy, interstitial fibrosis, accumulation of extracellular matrix proteins, and a decline in GFR lead to renal dysfunction and end-organ damage (Qian et al., 2008; Reiniger et al., 2010; Patel et al., 2016). TGF- $\beta 1$ plays a major role in the pathogenesis of interstitial fibrosis and glomerulosclerosis. Other studies have also revealed that ET-1 plays an important role in mediating fibrosis in various disease pathologies including hypertension and diabetes (Widyantoro et al., 2010; Wang et al., 2015), and that augmented ET-1 induced an increase in extracellular matrix protein $\mathrm{FN}-1$ production in the kidney in diabetes (Xu et al., 2008). Indeed, in this study we found that fa/fa rats on a high-salt diet had increased 

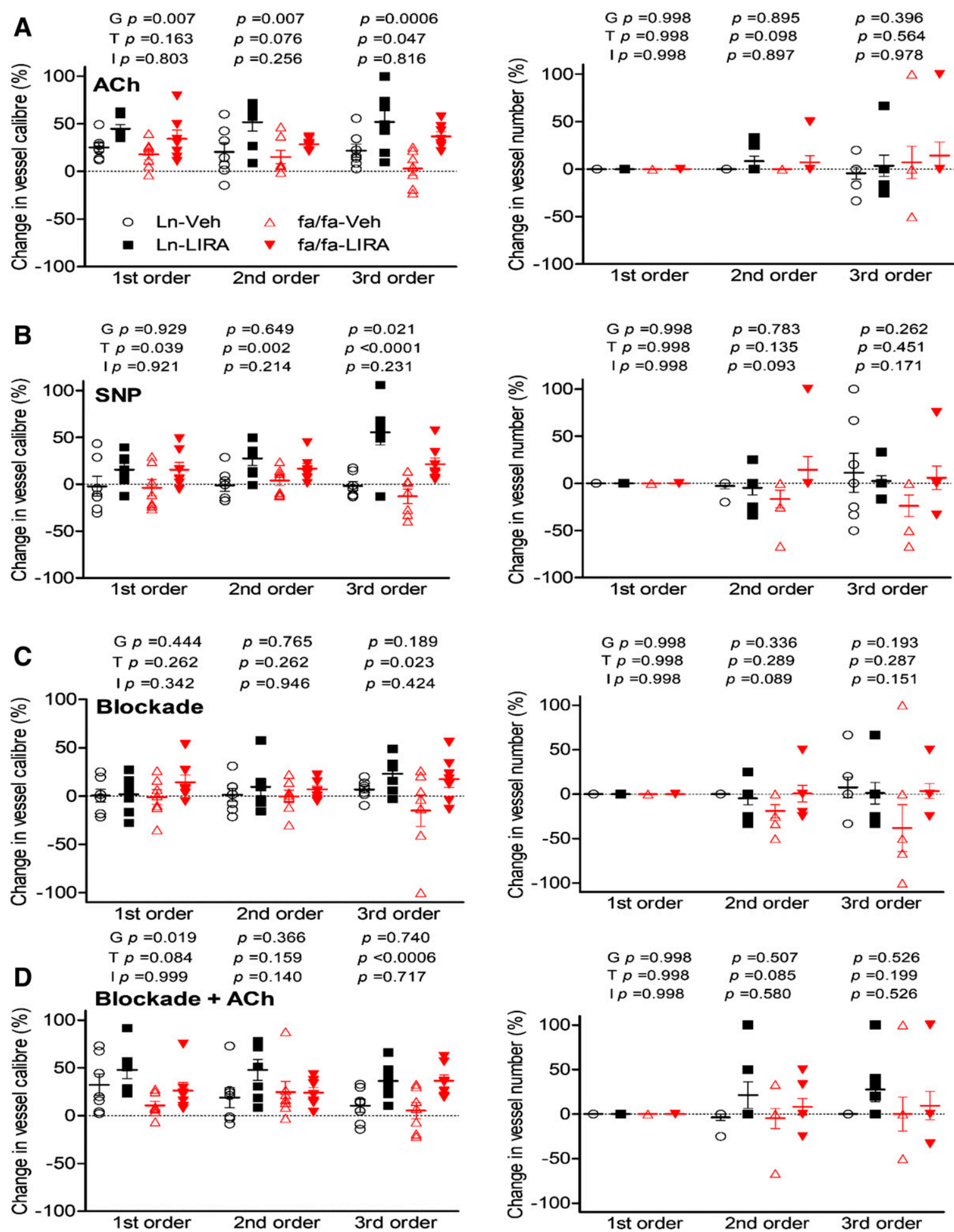

Fig. 7. LIRA treatment improved the capacity to increase perfused segments by NO-mediated dilation in Zucker fa/fa and Ln rats. (A-D) Percentage change in first- to third-order vessel caliber and visible vessel number during infusions of ACh, SNP, and ACh stimulation during the blockade of prostaglandin production and uncoupling of gap junctions from baseline. Mean \pm S.E.M.; $N=7$ rats per group. The significance of group differences was determined by two-way ANOVA for factors of genotype (G) and drug treatment (T) and their interaction (I).

expression of mRNA for TGF- $\beta 1$, ET-1, NADPH oxidase 1 , and Vcam-1 relative to lean fa/+ rats (Fig. 2). Previously, it was shown that treatment with a GLP-1R agonist inhibited the upregulation of TGF- $\beta 1$ in human mesangial cells in vitro (Li et al., 2012). However, treatment of Zucker rats with LIRA in this study did not result in significant reductions in renal mRNA expression of TGF- $\beta 1$, ET- 1 , NADPH oxidase 1 , or FN-1, and protein expression of TGF- $\beta 1$ was not significantly altered (Figs. 2 and 3D). On the other hand, endothelial function in the kidney was improved by LIRA, as indicated by the aforementioned improved vasodilator responses and substantially increased expression of eNOS protein (Fig. 3E), 
A

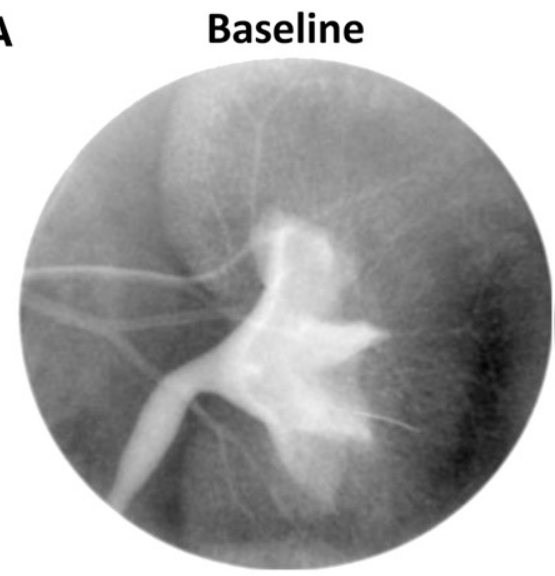

B

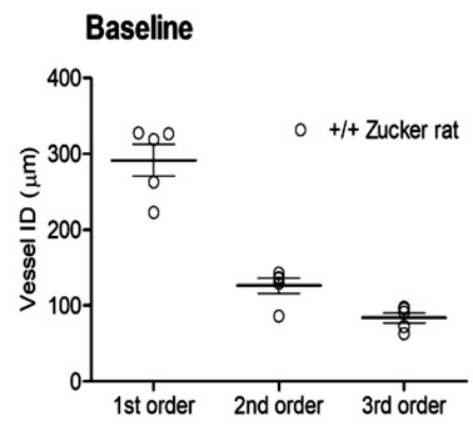

D

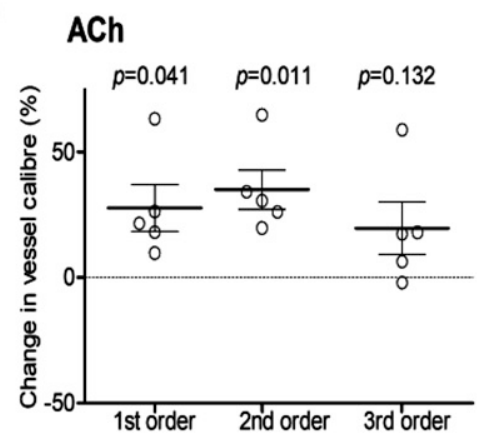

E

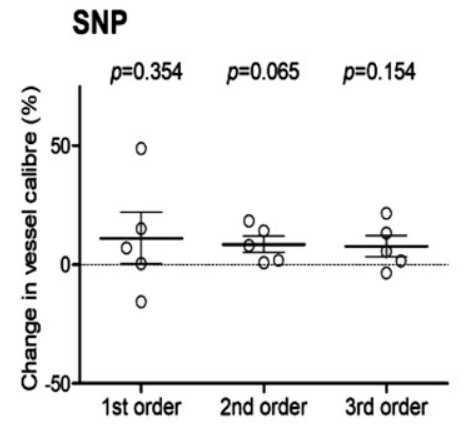

ACh

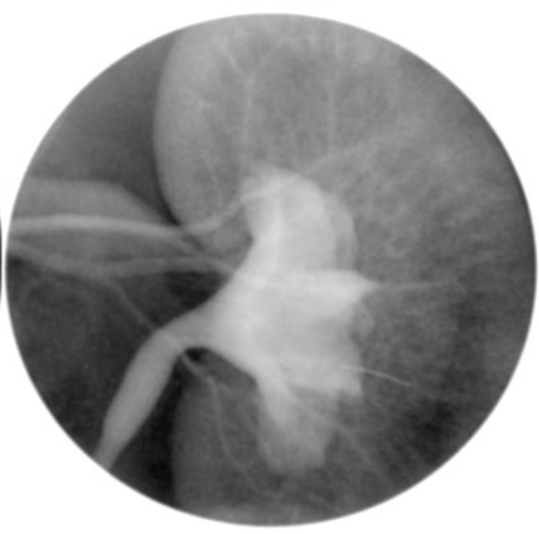

C
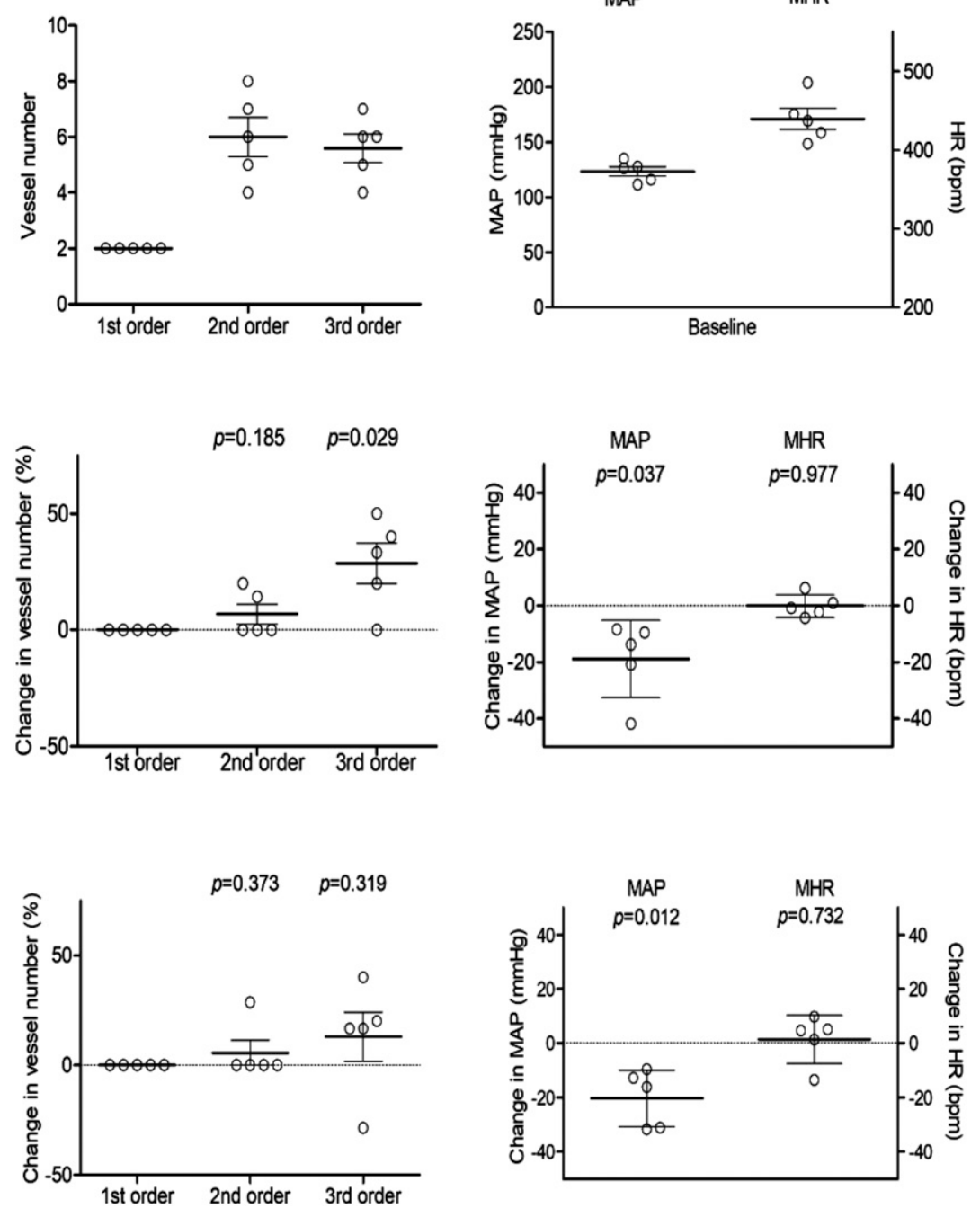

SNP
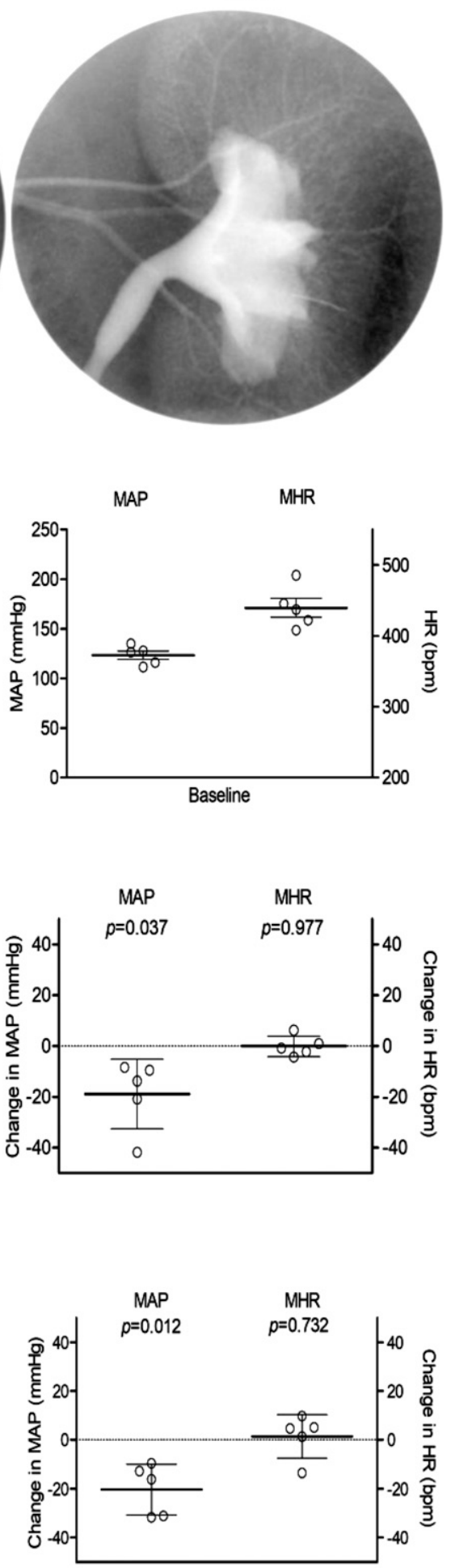

Fig. 8. Representative X-ray angiograms of the renal vasculature in 9-week-old Zucker rats on a normal-salt diet. (A) Images during baseline, infusions of ACh and SNP. (B) Vessel internal diameter and number categorized by branching order during baseline Ringer's lactate infusion. (C) MAP and mean heart rate (MHR) during baseline. (D and E) Percentage change in first- to third-order vessel caliber, visible vessel number, MAP, and MHR during infusions of ACh and SNP from baseline. Mean \pm S.E.M. $N=5$ rats per branching order. The significance was determined by column statistics.

and a strong trend toward reduced endothelial activation, as indicated by Vcam-1 mRNA expression. The latter findings are in line with a previous study, which revealed that
LIRA treatment improved renal injury by eNOS activation via inhibition of the nuclear factor kappa B pathway in streptozotocin-induced diabetic rats (Zhou et al., 2014). 
Based on the lack of effect of LIRA on TGF- $\beta 1$ and ET-1 signaling in this study, the modest amelioration of renal dysfunction and end-organ damage in the fa/fa rats on the high-salt diet by LIRA treatment suggests that this partial amelioration is attributable to improvements in renal microvessel endothelial function and normalization of renal vasomotor tone (hypothesized mechanism shown in Fig. 9). Partial reductions in nephrosclerosis, BUN, and urinary albumin elevation were associated with reduced macrophage infiltration of the nephrons and CD-68-positive protein expression (Figs. 3, A and C, and 4, A-D). Unlike the results in a previous study (Fujita et al., 2014), we did not observe a large reduction by LIRA in urinary albumin, albumin/creatinine ratio, and body weight as reported for obese diabetic mice on a normal $\operatorname{diet}$ (Fig. 1, D and E). This difference between studies might be in part due to the lower dose of LIRA administered in this study and the extra renal challenge presented by chronic exposure to a high-salt diet.

Sanders $(2009 a, b)$ showed that in salt sensitivity, high-salt intake stimulates the production of vasoconstrictors TGF- $\beta 1$ and ET-1, which evokes ROS generation (Fig. 9). Furthermore, it is well established that increased ROS generation in endothelial and vascular smooth muscle cells contributes to a state of oxidative stress in hypertension and diabetes (Li et al., 2003; Virdis et al., 2011; Nagasu et al., 2016). NADPH oxidase activation is triggered by the upregulation of vasoconstrictors including angiotensin-II, norepinephrine, and ET-1 (Zweier et al., 2011). Other studies implicate Nox1 in vascular inflammation, since oxidase activity and Nox1 expression are increased in smooth muscle cells and aortas of mice and rats with experimentally induced diabetes (Wendt et al., 2005; San Martin et al., 2007a,b). In addition, it has been reported that a high-salt diet caused accumulation of oxidative and nitrosative injury in renal tissues of Zucker obese rats (Patel et al., 2016). Even though it has been suggested that LIRA inhibits renal NADPH oxidase in diabetes models (Fujita et al., 2014; Herrera Pérez et al., 2016), it is somewhat surprising that we found no effect of LIRA on the expression of genes associated with elevation of oxidative stress and that renal NT protein did not differ significantly between rat groups (Figs. 2, B and E, and $3, \mathrm{~A}$ and $\mathrm{B})$. Therefore, we suggest here that direct inhibition of oxidative stress signaling is unlikely to be the main renoprotective action of LIRA in metabolic syndrome.

In this present study, we used a $1-\mu \mathrm{m}$ microfocus X-ray system to visualize the renal vasculature and investigate endothelial function in anesthetized Zucker rats. In vivo studies, including our own, have demonstrated that ACh-induced endothelium-dependent vasodilation is impaired in diabetes and high-salt diet animal models (DuPont et al., 2013; Pearson et al., 2013; Sonobe et al., 2015). Microangiography revealed that impairment in small artery/arteriole vasodilation was prevented in LIRA-treated rats, and more microvessels were perfused at baseline in fa/fa-LIRA group rats than in fa/fa-Veh rats (Fig. 5). Endothelium-dependent vasodilatory responses were most impaired in the small arteries and large arterioles in fa/fa-Veh group rats. Notably, using direct visualization of the renal vasculature we showed that smooth muscle dysfunction was also evident in the smaller vessels of fa/fa-Veh group rats, most likely due to upregulation of TGF- $\beta 1$, oxidative stress, and downregulation of soluble guanylyl cyclase in the renal vasculature (Du and Roberts, 2019), and this was prevented by concurrent treatment with LIRA. NO and or EDHF-mediated dilation in both conduit and resistance vessels was maintained by chronic LIRA treatment in the fa/fa rats. Only in the vehicle-treated fa/fa rats did the renal vessels become more constricted following blockade of COX and uncoupling of myoendothelial gap junctions. Gap junctions including connexins and various
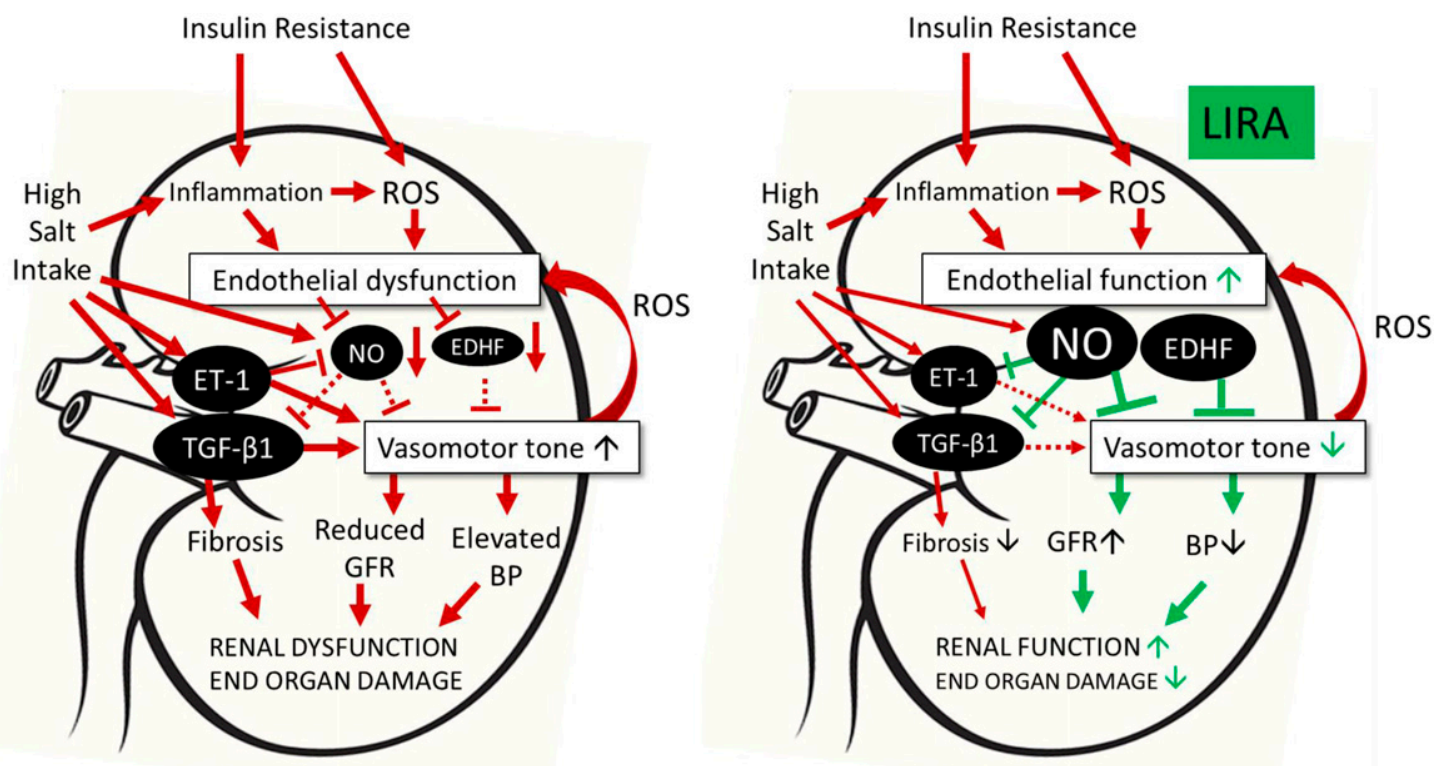

Fig. 9. Hypothesized protective effects of low-dose LIRA in renal dysfunction associated with the additive effects of obesity, insulin resistance, and highsalt diet. High-salt diet-induced renal dysfunction is characterized by upregulation of COX-2 and associated increase in renal vasoconstrictor tone and ROS production, endothelial dysfunction, and reduced eNOS, leading to elevated blood pressure and reduced GFR. LIRA treatment improved endothelial function and reduced renal vasomotor tone through the upregulation of eNOS and endothelium-dependent vasodilation (see Discussion for a detailed explanation). 
potassium channels are more important in longitudinal or conduced dilation (Feher et al., 2014), which increases the perfusion of side branches and more distal segments, rather than radial transmission of the hyperpolarization from the endothelium to the smooth muscle wall through myoendothelial gap junctions. Therefore, we assume the observed constriction of vessels that were visibly perfused throughout the imaging protocol, which was evoked by blockade in this study, is due to the inhibition of COX in the vasculature rather than inhibition of the myoendothelial gap junctions. This would imply that dilator prostanoids contribute significantly to the maintenance of renal perfusion in Zucker fa/fa rats exposed to high-salt intake, possibly to compensate for reduced NO bioavailability, impaired NO-mediated signaling, and/or increased prostanoids. Our findings suggest that the upregulation of inducible isoform COX-2 in fa/fa-Veh rats is associated with production of dilator prostanoids based on the changes in perfusion following nonselective COX inhibition. However, both COX-1 and COX-2 produce dilator and constrictor prostanoids. Upregulation and overactivation of COX-2 associated with vascular aging changes ACh-mediated relaxation to constriction (Guo et al., 2019). Furthermore, it has been demonstrated in the Zucker fa/fa rat that COX-2-derived prostanoids contribute to coronary constrictor responses mediated by oxidative stress (Santiago et al., 2016). Since ACh did not evoke constriction, and LIRA somewhat reduced COX-2 expression, there is no direct evidence at present to suggest COX-2-mediated renal constriction in our hypertensive Zucker fa/fa rats at this age. Previous studies have shown that EDHF-mediated dilation is increased in the Dahl salt-sensitive model rat and following chronic nitric oxide synthase inhibition (Goto et al., 2012; Simonet et al., 2012). However, in direct contrast to these findings, in the early stages of diabetes localized deficits in coronary EDHF-mediated dilation develop while NO-mediated dilation is still well maintained (Jenkins et al., 2012). A limitation of this study is that we did not attempt to establish if any of the known EDHF pathways are also impaired by high-salt intake and or metabolic syndrome, or indeed, if LIRA alters EDHFmediated dilation contributions in the kidney. In this study, LIRA did not appear to significantly modify conducted dilation within the kidney, which is largely mediated by various EDHFs. Recently, it has been demonstrated that GLP-1R agonists evoke endothelium-dependent dilation in isolated resistance arteries through both $\mathrm{NO}$ and EDHF $\left(\mathrm{Ca}^{2+}\right.$-activated potassium channels) signaling pathways (Bayram et al., 2014). We speculate that the upregulation of renal GLP-1R protein in fa/fa-Veh rats might be a compensatory mechanism to maintain renal perfusion in the face of diminished NO signaling since protein expression was normalized in association with NO-mediated dilation to SNP stimulation in fa/fa-LIRA rats. Importantly, such vasodilatory actions of LIRA provide further support for the hypothesized renoprotective actions of LIRA in this study (Fig. 9).

\section{Conclusions and Perspectives}

Taken together, our findings and the current literature suggest that high-salt diet induced renal dysfunction and injury occur through the upregulation of oxidative stress and inflammation, which subsequently lead to exacerbation of renal dysfunction in the presence of obesity (Fig. 9). In vivo renal angiograms revealed that LIRA treatment improved endothelium-dependent relaxation and restored renal blood flow in Zucker rat renal arteries, most likely through increased NO production rather than through an effect on TGF$\beta 1$ and ET-1 or oxidative stress and inflammation per se. Improvements in renal endothelial function and normalized blood pressure following LIRA treatment attenuated the progression of renal fibrosis and glomerular hypertrophy. However, further studies are warranted to determine if greater benefits in this salt-sensitive model of hypertension can be obtained with higher doses of LIRA.

\section{Authorship Contributions}

Participated in research design: Sukumaran, Shirai, Pearson. Conducted experiments: Sukumaran, Tsuchimochi, Sonobe.

Performed data analysis: Sukumaran.

Wrote or contributed to the writing of the manuscript: Sukumaran, Pearson.

\section{References}

Ali Q, Patel S, and Hussain T (2015) Angiotensin $\mathrm{AT}_{2}$ receptor agonist prevents saltsensitive hypertension in obese Zucker rats. Am $J$ Physiol Renal Physiol 308: F1379-F1385.

Ban K, Noyan-Ashraf MH, Hoefer J, Bolz SS, Drucker DJ, and Husain M (2008) Cardioprotective and vasodilatory actions of glucagon-like peptide 1 receptor are mediated through both glucagon-like peptide 1 receptor-dependent and -independent pathways. Circulation 117:2340-2350.

Bayram Z, Nacitarhan C, and Ozdem SS (2014) Effects of glucagon-like peptide-1 in diabetic rat small resistance arteries. J Cardiovasc Pharmacol 64:277-284.

Chen YC, Inagaki T, Fujii Y, Schwenke DO, Tsuchimochi H, Edgley AJ, Umetani K, Zhang Y, Kelly DJ, Yoshimoto M, et al. (2016) Chronic intermittent hypoxia accelerates coronary microcirculatory dysfunction in insulin-resistant Goto-Kakizaki rats. Am J Physiol Regul Integr Comp Physiol 311:R426-R439.

de Cavanagh EM, Ferder LF, Ferder MD, Stella IY, Toblli JE, and Inserra F (2010) Vascular structure and oxidative stress in salt-loaded spontaneously hypertensive rats: effects of losartan and atenolol. Am J Hypertens 23:1318-1325.

Du L and Roberts JD Jr. (2019) Transforming growth factor- $\beta$ downregulates sGC subunit expression in pulmonary artery smooth muscle cells via MEK and ERK signaling. Am J Physiol Lung Cell Mol Physiol 316:L20-L34.

DuPont JJ, Greaney JL, Wenner MM, Lennon-Edwards SL, Sanders PW, Farquhar WB, and Edwards DG (2013) High dietary sodium intake impairs endothelium-dependent dilation in healthy salt-resistant humans. J Hypertens 31:530-536.

Ellery SJ, Cai X, Walker DD, Dickinson H, and Kett MM (2015) Transcutaneous measurement of glomerular filtration rate in small rodents: through the skin for the win? Nephrology (Carlton) 20:117-123.

Eppel GA, Jacono DL, Shirai M, Umetani K, Evans RG, and Pearson JT (2009) Contrast angiography of the rat renal microcirculation in vivo using synchrotron radiation. Am J Physiol Renal Physiol 296:F1023-F1031.

Feher A, Broskova Z, and Bagi Z (2014) Age-related impairment of conducted dilation in human coronary arterioles. Am J Physiol Heart Circ Physiol 306: H1595-H1601.

Fujita H, Morii T, Fujishima H, Sato T, Shimizu T, Hosoba M, Tsukiyama K, Narita T, Takahashi T, Drucker DJ, et al. (2014) The protective roles of GLP-1R signaling in diabetic nephropathy: possible mechanism and therapeutic potential. Kidney Int 85:579-589.

Garg R, Williams GH, Hurwitz S, Brown NJ, Hopkins PN, and Adler GK (2011) Lowsalt diet increases insulin resistance in healthy subjects. Metabolism 60:965-968.

Goto K, Kansui Y, Oniki H, Ohtsubo T, Matsumura K, and Kitazono T (2012) Upregulation of endothelium-derived hyperpolarizing factor compensates for the loss of nitric oxide in mesenteric arteries of Dahl salt-sensitive hypertensive rats. Hypertens Res 35:849-854.

Guo Y, Xu C, Man AWC, Bai B, Luo C, Huang Y, Xu A, Vanhoutte PM, and Wang Y (2019) Endothelial SIRT1 prevents age-induced impairment of vasodilator responses by enhancing the expression and activity of soluble guanylyl cyclase in smooth muscle cells. Cardiovasc Res 115:678-690.

Hendarto H, Inoguchi T, Maeda Y, Ikeda N, Zheng J, Takei R, Yokomizo H, Hirata E, Sonoda N, and Takayanagi R (2012) GLP-1 analog liraglutide protects against oxidative stress and albuminuria in streptozotocin-induced diabetic rats via protein kinase A-mediated inhibition of renal $\mathrm{NAD}(\mathrm{P}) \mathrm{H}$ oxidases. Metabolism 61: 1422-1434.

Herrera Pérez Z, Weinfurter S, and Gretz N (2016) Transcutaneous assessment of renal function in conscious rodents. J Vis Exp 109:e53767.

Jenkins MJ, Edgley AJ, Sonobe T, Umetani K, Schwenke DO, Fujii Y, Brown RD, Kelly DJ, Shirai M, and Pearson JT (2012) Dynamic synchrotron imaging of diabetic rat coronary microcirculation in vivo. Arterioscler Thromb Vasc Biol 32: 370-377. 
Li L, Galligan JJ, Fink GD, and Chen AF (2003) Vasopressin induces vascular superoxide via endothelin-1 in mineralocorticoid hypertension. Hypertension 41: 663-668.

Li W, Cui M, Wei Y, Kong X, Tang L, and Xu D (2012) Inhibition of the expression of TGF- $\beta 1$ and CTGF in human mesangial cells by exendin-4, a glucagon-like peptide-1 receptor agonist. Cell Physiol Biochem 30:749-757.

Livak KJ and Schmittgen TD (2001) Analysis of relative gene expression data using real-time quantitative PCR and the $2^{-\Delta \Delta \mathrm{CT}}$ Method. Methods 25:402-408.

Maheshwari M, Romero CA, Monu SR, Kumar N, Liao TD, Peterson EL, and Carretero OA (2018) Renal protective effects of $N$-acetyl-seryl-aspartyllysyl-proline (Ac-SDKP) in obese rats on a high-salt diet. Am $J$ Hypertens $\mathbf{3 1}$ : 902-909.

Mann JFE, Ørsted DD, Brown-Frandsen K, Marso SP, Poulter NR, Rasmussen S, Tornøe K, Zinman B, and Buse JB; LEADER Steering Committee and Investigators (2017) Liraglutide and renal outcomes in type 2 diabetes. $N$ Engl $J$ Med 377: 839-848.

Meng XM, Huang XR, Chung AC, Qin W, Shao X, Igarashi P, Ju W, Bottinger EP, and Lan HY (2010) Smad2 protects against TGF- $\beta$ /Smad3-mediated renal fibrosis. $J$ Am Soc Nephrol 21:1477-1487.

Mima A, Hiraoka-Yamomoto J, Li Q, Kitada M, Li C, Geraldes P, Matsumoto M, Mizutani K, Park K, Cahill C, et al. (2012) Protective effects of GLP-1 on glomerular endothelium and its inhibition by $\operatorname{PKC} \beta$ activation in diabetes. Diabetes 61: $2967-2979$

Nagasu H, Satoh M, Kiyokage E, Kidokoro K, Toida K, Channon KM, Kanwar YS, Sasaki T, and Kashihara N (2016) Activation of endothelial NAD(P)H oxidase accelerates early glomerular injury in diabetic mice. Lab Invest 96:25-36.

Oh SW, Han KH, Han SY, Koo HS, Kim S, and Chin HJ (2015) Association of sodium excretion with metabolic syndrome, insulin resistance, and body fat. Medicine (Baltimore) 94:e1650.

Patel SN, Ali Q, and Hussain T (2016) Angiotensin II type 2-receptor agonist C21 reduces proteinuria and oxidative stress in kidney of high-salt-fed obese Zucker rats. Hypertension 67:906-915.

Pearson JT, Jenkins MJ, Edgley AJ, Sonobe T, Joshi M, Waddingham MT, Fujii Y, Schwenke DO, Tsuchimochi H, Yoshimoto M, et al. (2013) Acute Rho-kinase in hibition improves coronary dysfunction in vivo, in the early diabetic microcirculation. Cardiovasc Diabetol 12:111.

Qian Y, Feldman E, Pennathur S, Kretzler M, and Brosius FC III (2008) From fibrosis to sclerosis: mechanisms of glomerulosclerosis in diabetic nephropathy. Diabetes 57:1439-1445.

Quigley JE, Elmarakby AA, Knight SF, Manhiani MM, Stepp DW, Olearzcyk JJ, and Imig JD (2009) Obesity induced renal oxidative stress contributes to renal injury in salt-sensitive hypertension. Clin Exp Pharmacol Physiol 36: 724-728.

Rao A, Pandya V, and Whaley-Connell A (2015) Obesity and insulin resistance in resistant hypertension: implications for the kidney. Adv Chronic Kidney Dis 22 211-217.

Reiniger N, Lau K, McCalla D, Eby B, Cheng B, Lu Y, Qu W, Quadri N, Ananthakrishnan R, Furmansky M, et al. (2010) Deletion of the receptor for advanced glycation end products reduces glomerulosclerosis and preserves renal function in the diabetic OVE26 mouse. Diabetes 59:2043-2054.

Sanders PW (2009a) Dietary salt intake, salt sensitivity, and cardiovascular health. Hypertension 53:442-445.

Sanders PW (2009b) Vascular consequences of dietary salt intake. Am J Physio Renal Physiol 297:F237-F243.

San Martín A, Du P, Dikalova A, Lassègue B, Aleman M, Góngora MC, Brown K, Joseph G, Harrison DG, Taylor WR, et al. (2007a) Reactive oxygen species-selective regulation of aortic inflammatory gene expression in Type 2 diabetes. Am J Physiol Heart Circ Physiol 292:H2073-H2082.
San Martin A, Foncea R, Laurindo FR, Ebensperger R, Griendling KK, and Leighton F (2007b) Nox1-based NADPH oxidase-derived superoxide is required for VSMC activation by advanced glycation end-products. Free Radic Biol Med 42:1671-1679.

Santiago E, Martínez MP, Climent B, Muñoz M, Briones AM, Salaices M, GarcíaSacristán A, Rivera L, and Prieto D (2016) Augmented oxidative stress and preserved vasoconstriction induced by hydrogen peroxide in coronary arteries in obesity: role of COX-2. Br J Pharmacol 173:3176-3195.

Satoh M (2012) Endothelial dysfunction as an underlying pathophysiological condition of chronic kidney disease. Clin Exp Nephrol 16:518-521.

Shirai M, Schwenke DO, Tsuchimochi H, Umetani K, Yagi N, and Pearson JT (2013) Synchrotron radiation imaging for advancing our understanding of cardiovascular function. Circ Res 112:209-221.

Simonet S, Isabelle M, Bousquenaud M, Clavreul N, Félétou M, Vayssettes-Courchay $\mathrm{C}$, and Verbeuren TJ $(2012) \mathrm{K}_{\mathrm{Ca}} 3.1$ channels maintain endothelium-dependent vasodilatation in isolated perfused kidneys of spontaneously hypertensive rats after chronic inhibition of NOS. Br J Pharmacol 167:854-867.

Sonobe T, Tsuchimochi H, Schwenke DO, Pearson JT, and Shirai M (2015) Treadmill running improves hindlimb arteriolar endothelial function in type 1 diabetic mice as visualized by X-ray microangiography. Cardiovasc Diabetol 14:51.

Virdis A, Duranti E, and Taddei S (2011) Oxidative stress and vascular damage in hypertension: role of angiotensin II. Int $J$ Hypertens 2011:916310.

Wang X, Guo Z, Ding Z, Khaidakov M, Lin J, Xu Z, Sharma SG, Jiwani S, and Mehta JL (2015) Endothelin-1 upregulation mediates aging-related cardiac fibrosis. $J$ Mol Cell Cardiol 80:101-109.

Wei SY, Wang YX, Zhang QF, Zhao SL, Diao TT, Li JS, Qi WR, He YX, Guo XY, Zhang MZ, et al. (2017) Multiple mechanisms are involved in salt-sensitive hypertension-induced renal injury and interstitial fibrosis. Sci Rep 7:45952.

Wendt MC, Daiber A, Kleschyov AL, Mülsch A, Sydow K, Schulz E, Chen K, Keaney JF Jr, Lassègue B, Walter U, et al. (2005) Differential effects of diabetes on the expression of the gp91 ${ }^{\text {phox }}$ homologues nox1 and nox4. Free Radic Biol Med 39: 381-391.

Widyantoro B, Emoto N, Nakayama K, Anggrahini DW, Adiarto S, Iwasa N, Yagi K, Miyagawa K, Rikitake Y, Suzuki T, et al. (2010) Endothelial cell-derived endothelin-1 promotes cardiac fibrosis in diabetic hearts through stimulation of endothelial-to-mesenchymal transition. Circulation 121:2407-2418.

Wu MT, Lam KK, Lee WC, Hsu KT, Wu CH, Cheng BC, Ng HY, Chi PJ, Lee YT, and Lee CT (2012) Albuminuria, proteinuria, and urinary albumin to protein ratio in chronic kidney disease. J Clin Lab Anal 26:82-92.

Xu B, Chiu J, Feng B, Chen S, and Chakrabarti S (2008) PARP activation and the alteration of vasoactive factors and extracellular matrix protein in retina and kidney in diabetes. Diabetes Metab Res Rev 24:404-412.

Yuzawa Y, Niki I, Kosugi T, Maruyama S, Yoshida F, Takeda M, Tagawa Y, Kaneko Y, Kimura T, Kato N, et al. (2008) Overexpression of calmodulin in pancreatic beta cells induces diabetic nephropathy. J Am Soc Nephrol 19:1701-1711.

Zhou SJ, Bai L, Lv L, Chen R, Li CJ, Liu XY, Yu DM, and Yu P (2014) Liraglutide ameliorates renal injury in streptozotocin-induced diabetic rats by activating endothelial nitric oxide synthase activity via the downregulation of the nuclear factor- $\kappa$ B pathway. Mol Med Rep 10:2587-2594.

Zweier JL, Chen CA, and Druhan LJ (2011) S-glutathionylation reshapes our understanding of endothelial nitric oxide synthase uncoupling and nitric oxide/ reactive oxygen species-mediated signaling. Antioxid Redox Signal 14:1769-1775.

Address correspondence to: Dr. Vijayakumar Sukumaran, Department of Cardiac Physiology, National Cerebral and Cardiovascular Center Research Institute, Suita, Osaka 565-8565, Japan. E-mail: sukumaran.vijayakumar09@ ncvc.go.jp; svkumar79@gmail.com 